\title{
A FRUGAL FETI-DP AND BDDC COARSE SPACE FOR HETEROGENEOUS PROBLEMS*
}

\author{
ALEXANDER HEINLEIN $^{\dagger \ddagger}$, AXEL KLAWONN ${ }^{\dagger \ddagger}$, MARTIN LANSER $^{\dagger \ddagger}$, AND JANINE WEBER ${ }^{\dagger}$
}

\begin{abstract}
The convergence rate of domain decomposition methods is generally determined by the eigenvalues of the preconditioned system. For second-order elliptic partial differential equations, coefficient discontinuities with a large contrast can lead to a deterioration of the convergence rate. Only by implementing an appropriate coarse space, or second level, a robust domain decomposition method can be obtained. In this article, a new frugal coarse space for FETI-DP (Finite Element Tearing and Interconnecting-Dual Primal) and BDDC (Balancing Domain Decomposition by Constraints) methods is presented, which has a lower set-up cost than competing adaptive coarse spaces. In particular, in contrast to adaptive coarse spaces, it does not require the solution of any local generalized eigenvalue problems. The approach considered here aims at a low-dimensional approximation of the adaptive coarse space by using appropriate weighted averages, and it is robust for a broad range of coefficient distributions for diffusion and elasticity problems. However, in general, for completely arbitrary coefficient distributions with high contrast, some additional, adaptively chosen constraints are necessary in order to guarantee robustness. In this article, the robustness is heuristically justified as well as numerically shown for several coefficient distributions. The new coarse space is compared to adaptive coarse spaces, and parallel scalability up to 262144 parallel cores for a parallel BDDC implementation with the new coarse space is shown. The superiority of the new coarse space over classic coarse spaces with respect to parallel weak scalability and time-to-solution is confirmed by numerical experiments. Since the new frugal coarse space is computationally inexpensive, it could serve as a new default coarse space, which, for very challenging coefficient distributions, could then still be enhanced by adaptively chosen constraints.
\end{abstract}

Key words. FETI-DP, BDDC, robust coarse spaces, adaptive domain decomposition methods

AMS subject classifications. 68W10, 65N22, 65N55, 65F08, 65F10, 65Y05

1. Introduction. Domain decomposition methods are robust and parallel scalable iterative solvers for large systems of equations arising from the discretization of partial differential equations, e.g., by finite elements. In general, the computational domain is decomposed into a number of overlapping or nonoverlapping subdomains. Here, we focus on two classes of nonoverlapping domain decomposition methods, namely FETI-DP (Finite Element Tearing and Interconnecting-Dual Primal) $[15,16,46,48]$ and BDDC (Balancing Domain Decomposition by Constraints) $[10,11,50,52,53]$ methods. Both algorithms have been successfully applied to a wide range of model problems and have been shown to be parallel scalable for up to hundreds of thousands of compute cores [2, 3, 34, 35, 36, 37, 43, 66]. In general, domain decomposition methods obtain their robustness and parallel scalability from an appropriate coarse space, i.e., a second level. For nonoverlapping domain decomposition methods, such a coarse space can be constructed by simply sub-assembling the system in selected primal variables using geometric information. For these classic coarse spaces, condition number bounds have been proven for a wide range of model problems [42, 45, 46, 48, 56]. However, the respective condition number bounds are only valid under certain restrictive assumptions on the coefficient functions of the differential equation considered, e.g., the diffusion coefficient in case of a diffusion problem or the Young modulus in case of a linear elasticity problem.

*Received November 30, 2019. Accepted November 23, 2020. Published online on December 21, 2020. Recommended by Marco Donatelli. This work was supported in part, as project EXASTEEL, by Deutsche Forschungsgemeinschaft (DFG) through the Priority Programme 1648 "Software for Exascale Computing" (SPPEXA) under grant number 230723766.

${ }^{\dagger}$ Department of Mathematics and Computer Science, University of Cologne, Weyertal 86-90, 50931 Köln (\{alexander.heinlein, axel.klawonn, martin.lanser, janine.weber\}@uni-koeln.de) http://www. numerik.uni-koeln.de.

${ }^{\ddagger}$ Center for Data and Simulation Science, University of Cologne http://www.cds.uni-koeln.de. 


\section{ETNA}

Kent State University and

Johann Radon Institute (RICAM)

For more general and complex coefficient functions with arbitrary jumps along or across the interface, the classic condition number bounds do not hold anymore, and the convergence rate of the classic domain decomposition methods typically deteriorates. Thus, different adaptive coarse space techniques for several domain decomposition methods have been developed within the recent years to cope with heterogeneous coefficient functions with large jumps; see, e.g., $[5,7,9,13,14,17,18,19,23,24,25,30,31,32,33,39,40,54,55,57,58,61,62]$. Most of these methods rely on the solution of certain local generalized eigenvalue problems and use selected eigenvectors to enhance the coarse space. By including these adaptive coarse spaces, the algorithm is again robust with respect to discontinuous coefficient functions for both diffusion and elasticity problems. In particular, contrast independent condition number estimates can be proven for most of these adaptive coarse spaces. As a drawback in a parallel implementation, the set-up and the solution of the eigenvalue problems take up a significant amount of time. In [27], we have introduced the concept of training a neural network to make an automatic decision on which parts of the interface, for two-dimensional model problems, i.e., on which edges, the solution of the eigenvalue problem is indeed necessary to obtain a robust algorithm. This can reduce the time-to-solution significantly.

A further important experimental observation is that for many realistic coefficient distributions often only a small number of jumps with respect to a specific edge or face occurs for a large number of edges and faces. Thus, for many edges and faces, the computation of all eigenvectors is indeed unnecessary since already a single or a small number of constraints is sufficient for robustness on these edges and faces. In the present paper, we introduce an alternative and more frugal approach. In principle, we aim to compute a low-dimensional approximation of the adaptive coarse space by constructing weighted averages along edges or faces. Earlier works [22, 24, 49] showed that heuristic coarse spaces, which approximate adaptive coarse spaces and do not require the solution of local generalized eigenvalue problems, can be constructed for overlapping Schwarz domain decomposition methods. Further approaches to build nonoverlapping domain decomposition methods that are robust for a broader range of coefficient distributions are presented in [1], where the domain is decomposed based on the coefficient distribution, and in [4], where deluxe scaling is introduced to obtain a more robust BDDC or FETI-DP method. In [20, 21] a robust coarse space for almost incompressible elasticity problems for the FETI-DP method is suggested. Here, using our experience with adaptive coarse spaces, we construct constraints in a similar approach. We will observe that for many realistic coefficient distributions, the resulting constraints are already sufficient for fast convergence. The approach presented here can further be interpreted as a generalization of the classic weighted averages over edges as introduced in [42]. In fact, for the case of discontinuities which are aligned with the interface, our new constraints enforce comparable constraints as those classic weighted averages. However, our new weighted constraints additionally lead to robust algorithms in more general and complex cases of discontinuities not aligned with the interface. In general, for completely arbitrary coefficient jumps, additional constraints, which are obtained by adaptive coarse spaces, may be necessary to further improve the convergence of the algorithm and guarantee robustness with respect to the coefficient contrast.

To provide a brief impression of the capability of our proposed coarse space, we consider a simple exemplary coefficient distribution with coefficient jumps as in Figure 3.3 (left). In Table 1.1, we compare numerical results for the so-called standard approaches, i.e., the classic weighted edge averages [42], an adaptive coarse space variant [54, 55], and our proposed frugal coarse space. While our approach is competitive with the adaptive approach, the one with classic weighted edge averages clearly fails to provide a robust algorithms, although the dimension of the classic coarse space is three times larger. Notably, in contrast to the adaptive constraints, the construction of our new constraints is easily parallelizable and fairly cheap, 
TABLE 1.1

Dimensions of the coarse space (\# c.), condition numbers (cond), and iteration numbers (it) for the FETI-DP algorithm for a stationary diffusion problem on the unit square with $4 \times 4$ subdomains for the coefficient distribution as in Figure 3.3 (left). Homogeneous Dirichlet boundary conditions are set on the left-hand side of the unit square. The larger coefficient is $1 \mathrm{e} 6$, and the smaller coefficient is 1.

\begin{tabular}{c|ccc|ccc||crc}
\multicolumn{3}{|c|}{ adaptive } & \multicolumn{3}{c||}{ classic weighted avg. } & \multicolumn{3}{c}{ new approach } \\
$\mathrm{H} / \mathrm{h}$ & \# c. & cond & it & \# c. & cond & it & \# c. & cond & it \\
\hline \hline \multicolumn{8}{c}{ stationary diffusion } \\
\hline 8 & 4 & 3.60 & 12 & 12 & 61559.3 & 20 & 4 & 3.60 & 12 \\
16 & 4 & 3.95 & 13 & 12 & 99656.1 & 24 & 4 & 3.96 & 13 \\
32 & 4 & 5.02 & 15 & 12 & $1.1775 \mathrm{e} 05$ & 26 & 4 & 5.04 & 15
\end{tabular}

i.e., the resulting coarse space can be computed with less effort than a few CG (conjugate gradient) iterations since it does not require the solution of any eigenvalue problems. Thus, our new approach could be implemented as a default rule to enhance the coarse space when no implementation of adaptive coarse space techniques is available or the time-to-solution should be reduced. Our numerical experiments show that our new frugal approach leads to a robust algorithm for problems with a realistic coefficient distribution and can especially outperform classic edge and face averages in cases of complex coefficient functions.

The remainder of the paper is organized as follows. In Section 2, we first introduce the model problems and the necessary notation to outline our domain decomposition methods. We then describe both the FETI-DP and the BDDC algorithm in more detail. In Section 3, we give a detailed description of our new constraints. For the convenience of the reader, we first explain the construction for diffusion problems, which is the simpler case. For the case of linear elasticity, we then give the corresponding formulae based on weighted rigid body modes. At first, in Section 4, we provide serial results by applying the proposed approach to different coefficient distributions, proving that our algorithm is robust. Finally, in Section 5, we present results comparing our new approach to classic averages using our parallel BDDC implementation applied to difficult model problems. For all our numerical tests, we consider stationary linear diffusion and linear elasticity problems.

2. Algorithms and model problems. As a model problem, we consider both stationary linear diffusion problems as well as linear elasticity problems in two and three dimensions. We will focus on highly heterogeneous problems with large discontinuities in the material stiffness or the diffusion coefficient, respectively. For the remainder of this section, we denote by $d=2,3$ the dimension of our domain $\Omega \subset \mathbb{R}^{d}$.

2.1. Stationary diffusion. As a first model problem, we consider a stationary diffusion problem in its variational form with various coefficient functions $\rho: \Omega \rightarrow \mathbb{R}$, which may have large jumps. We assume that one part of the boundary of the domain, $\partial \Omega_{D}$, has homogeneous Dirichlet boundary conditions, while $\partial \Omega_{N}:=\partial \Omega \backslash \partial \Omega_{D}$ has a natural boundary condition $\frac{\partial u}{\partial x}=g$. Throughout this paper, we only consider a homogeneous flow $g=0$. Thus, the model problem can be written as: Find $u \in H_{0}^{1}\left(\Omega, \partial \Omega_{D}\right):=\left\{u \in H^{1}(\Omega): u=0\right.$ on $\left.\partial \Omega_{D}\right\}$, such that

$$
\int_{\Omega} \rho \nabla u \cdot \nabla v d x=\int_{\Omega} f v d x, \quad \forall v \in H_{0}^{1}\left(\Omega, \partial \Omega_{D}\right) .
$$

Concrete examples of different coefficient functions $\rho$ as well as concrete choices of boundary conditions are given in detail in Section 4. 
2.2. Linear elasticity. We consider an elastic body $\Omega \subset \mathbb{R}^{d}, d=2,3$. We denote by $\mathbf{u}: \Omega \rightarrow \mathbb{R}^{d}$ the displacement of the body, by $f$ a given volume force, and by $g$ a given surface force to the body, respectively. Here, we only consider a homogeneous surface force $g=0$.

We introduce the vector-valued Sobolev space $\mathbf{H}_{0}^{1}\left(\Omega, \partial \Omega_{D}\right):=\left(H_{0}^{1}\left(\Omega, \partial \Omega_{D}\right)\right)^{d}$. The problem of linear elasticity consists in finding the displacement $\mathbf{u} \in \mathbf{H}_{0}^{1}\left(\Omega, \partial \Omega_{D}\right)$ such that

$$
\int_{\Omega} G \varepsilon(\mathbf{u}): \varepsilon(\mathbf{v}) d \mathbf{x}+\int_{\Omega} G \beta \operatorname{div} \mathbf{u} \operatorname{div} \mathbf{v} d \mathbf{x}=\langle\mathbf{F}, \mathbf{v}\rangle,
$$

for all $\mathbf{v} \in \mathbf{H}_{0}^{1}\left(\Omega, \partial \Omega_{D}\right)$ for given material functions $G: \Omega \rightarrow \mathbb{R}$ and $\beta: \Omega \rightarrow \mathbb{R}$ and for the right-hand side

$$
\langle\mathbf{F}, \mathbf{v}\rangle=\int_{\Omega} \mathbf{f}^{T} \mathbf{v} d \mathbf{x} .
$$

The material parameters $G$ and $\beta$ depend on the Young modulus $E>0$ and the Poisson ratio $\nu \in(0,1 / 2)$ by $G=E /(1+\nu)$ and $\beta=\nu /(1-2 \nu)$. Here, we restrict ourselves to compressible linear elasticity; hence the Poisson ratio $\nu$ is bounded away from $1 / 2$. Furthermore, the linearized strain tensor $\varepsilon=\left(\varepsilon_{i j}\right)_{i j}$ is defined by $\varepsilon_{i j}(\mathbf{u}):=\frac{1}{2}\left(\frac{\partial u_{i}}{\partial x_{j}}+\frac{\partial u_{j}}{\partial x_{i}}\right)$, and we introduce the notation

$$
\varepsilon(\mathbf{u}): \varepsilon(\mathbf{v}):=\sum_{i, j=1}^{d} \varepsilon_{i j}(\mathbf{u}) \varepsilon_{i j}(\mathbf{v}), \quad(\varepsilon(\mathbf{u}), \varepsilon(\mathbf{v}))_{L_{2}(\Omega)}:=\int_{\Omega} \varepsilon(\mathbf{u}): \varepsilon(\mathbf{v}) d \mathbf{x} .
$$

The corresponding bilinear form associated with linear elasticity can now be written as

$$
a(\mathbf{u}, \mathbf{v})=(G \varepsilon(\mathbf{u}), \varepsilon(\mathbf{v}))_{L_{2}(\Omega)}+(G \beta \operatorname{div} \mathbf{u}, \operatorname{div} \mathbf{v})_{L_{2}(\Omega)} .
$$

Since we will only consider compressible elastic materials, it is sufficient to discretize our elliptic problem by low-order conforming finite elements, e.g., linear or trilinear elements.

\subsection{The FETI-DP and the BDDC algorithm.}

2.3.1. Domain decomposition. Let us briefly describe the preliminaries for our domain decomposition methods to introduce the FETI-DP and BDDC algorithms. For a given domain $\Omega \subset \mathbb{R}^{d}, d=2,3$, we assume a decomposition into $N \in \mathbb{N}$ nonoverlapping subdomains $\Omega_{i}$, $i=1, \ldots, N$, such that $\bar{\Omega}=\bigcup_{i=1}^{N} \bar{\Omega}_{i}$. We presume that each of the subdomains $\Omega_{i}$ is the union of finite elements such that we have matching finite element nodes on the interface $\Gamma:=\left(\bigcup_{i=1}^{N} \partial \Omega_{i}\right) \backslash \partial \Omega$. In our case, each subdomain is the union of shape-regular elements of diameter $\mathcal{O}(h)$. The diameter of a subdomain $\Omega_{i}$ is denoted by $H_{i}$ or, generically, by $H=\max _{i}\left(H_{i}\right)$. We denote by $W_{i}$ the local finite element space associated with $\Omega_{i}$. In case of a two-dimensional domain $\Omega \subset \mathbb{R}^{2}$, the finite element nodes on the interface are either vertex nodes, belonging to the boundary of more than two subdomains, or edge nodes, belonging to the boundary of exactly two subdomains. For the case of a three-dimensional domain $\Omega \subset \mathbb{R}^{3}$, edge nodes also belong to the boundary of more than two subdomains, and the interface further consists of face nodes, belonging to the boundary of exactly two subdomains; see, e.g., [41, Def. 2.1 and Def. 2.2] and [46, Def. 3.1]. All finite element nodes inside a subdomain $\Omega_{i}$ are denoted as interior nodes. For a given domain decomposition, we obtain the local finite element problems $K^{(i)} u^{(i)}=f^{(i)}$ with $K^{(i)}: W_{i} \rightarrow W_{i}$ and $f^{(i)} \in W_{i}$ by restricting the considered differential equation (see Sections 2.1 and 2.2) to $\Omega_{i}$ and discretizing its variational formulation in the finite element space $W_{i}$. Let us remark that 
the matrices $K^{(i)}$ are, in general, not invertible for those subdomains that have no contact to the Dirichlet boundary.

We define the product space $W:=\prod_{i=1}^{N} W_{i}$ and denote by $\widehat{W} \subset W$ the space of functions in $W$ that are continuous on $\Gamma$. For FETI-DP and BDDC, we partition the finite element variables $u^{(i)} \in W_{i}$ into interior variables $u_{I}^{(i)}$ and, on the interface, into dual variables $u_{\Delta}^{(i)}$ and primal variables $u_{\Pi}^{(i)}$. We denote the respective degrees of freedom by the indizes $I, \Delta$, and $\Pi$. In the present article, we always choose all variables belonging to vertices as primal variables. Thus, the dual variables always belong to edges and/or faces. Note that other choices are possible. Finally, we introduce the space $\widetilde{W}$, consisting of functions $w \in W$ that are continuous in the primal variables. We thus have $\widehat{W} \subset \widetilde{W} \subset W$.

2.3.2. Standard FETI-DP. As a first step for both the FETI-DP $[15,16]$ and the BDDC $[11,52]$ algorithms, we compute the local stiffness matrices $K^{(i)}$ and the local righthand sides $f^{(i)}$ for every subdomain $\Omega_{i}, i=1, \ldots, N$. The local problems are completely decoupled, and, as already mentioned, the matrices $K^{(i)}$ are, in general, not invertible for subdomains without contact to the Dirichlet boundary. Both the FETI-DP and the BDDC algorithms deal with this difficulty by sub-assembling the decoupled system in selected primal variables $\Pi$.

Let us first introduce the simple restriction operators $R_{i}: V^{h} \rightarrow W_{i}, i=1, \ldots, N$, the block vectors $u^{T}:=\left(u^{(1) T}, \ldots, u^{(N) T}\right)$ and $f^{T}:=\left(f^{(1) T}, \ldots, f^{(N) T}\right)$, and the block matrices $R^{T}:=\left(R_{1}^{T}, \ldots, R_{N}^{T}\right)$ and $K=\operatorname{diag}\left(K^{(1)}, \ldots, K^{(N)}\right)$. We then obtain the fully assembled system

$$
K_{g}=R^{T} K R
$$

and the fully assembled right-hand side

$$
f_{g}=R^{T} f
$$

The block matrix $K$ is not invertible as long as a single subdomain has no contact to the Dirichlet boundary. Thus, the system $K u=f$ has no unique solution, i.e., an unknown vector $u$ might be discontinuous on the interface. Let us now describe how the continuity of $u \in W:=W_{1} \times \ldots \times W_{N}$ on the interface is enforced using FETI-DP. Here, we use a presentation of the FETI-DP method which is very similar to the compact notation in [40].

We assume the following partitioning of the local stiffness matrices $K^{(i)}$, the local load vectors $f^{(i)}$, and the local solutions $u^{(i)}$ using a subdivision of the degrees of freedom as introduced in Section 2.3.1:

$$
K^{(i)}=\left[\begin{array}{ccc}
K_{I I}^{(i)} & K_{\Delta I}^{(i) T} & K_{\Pi I}^{(i) T} \\
K_{\Delta I}^{(i)} & K_{\Delta \Delta}^{(i)} & K_{\Pi \Delta}^{(i) T} \\
K_{\Pi I}^{(i)} & K_{\Pi \Delta}^{(i)} & K_{\Pi \Pi}^{(i)}
\end{array}\right], \quad u^{(i)}=\left[\begin{array}{c}
u_{I}^{(i)} \\
u_{\Delta}^{(i)} \\
u_{\Pi}^{(i)}
\end{array}\right], \quad \text { and } \quad f^{(i)}=\left[\begin{array}{c}
f_{I}^{(i)} \\
f_{\Delta}^{(i)} \\
f_{\Pi}^{(i)}
\end{array}\right] .
$$

It is often convenient to further introduce the union of interior and dual degrees of freedom as an additional set of degrees of freedom denoted by the index $B$. This leads to a more compact notation, and we can define the following matrices and vectors:

$$
K_{B B}^{(i)}=\left[\begin{array}{cc}
K_{I I}^{(i)} & K_{\Delta I}^{(i) T} \\
K_{\Delta I}^{(i)} & K_{\Delta \Delta}^{(i)}
\end{array}\right], \quad K_{\Pi B}^{(i)}=\left[\begin{array}{cc}
K_{\Pi I}^{(i)} & K_{\Pi \Delta}^{(i)}
\end{array}\right], \quad \text { and } \quad f_{B}^{(i)}=\left[\begin{array}{ll}
f_{I}^{(i) T} & f_{\Delta}^{(i) T}
\end{array}\right]^{T} .
$$

We then introduce the block diagonal matrices

$$
\begin{aligned}
& K_{B B}=\operatorname{diag}_{i=1}^{N} K_{B B}^{(i)}, \quad K_{I I}=\operatorname{diag}_{i=1}^{N} K_{I I}^{(i)}, \\
& K_{\Delta \Delta}=\operatorname{diag}_{i=1}^{N} K_{\Delta \Delta}^{(i)}, \quad \text { and } \quad K_{\Pi \Pi}=\operatorname{diag}_{i=1}^{N} K_{\Pi \Pi}^{(i)} .
\end{aligned}
$$


Analogously, we obtain the block vector $u_{B}=\left[u_{B}^{(1) T}, \ldots, u_{B}^{(N) T}\right]^{T}$ and the block righthand side $f_{B}=\left[f_{B}^{(1) T}, \ldots, f_{B}^{(N) T}\right]^{T}$. For the FETI-DP algorithm, continuity in the primal variables $\Pi$ is enforced by a finite element assembly process, while continuity in the dual variables $\Delta$ is enforced iteratively by Lagrangian multipliers $\lambda$. To describe the primal assembly process, we introduce the assembly operators $R_{\Pi}^{(i)^{T}}$, which is a matrix consisting of values in $\{0,1\}$. This yields the primally assembled matrices

$$
\widetilde{K}_{\Pi \Pi}=\sum_{i=1}^{N} R_{\Pi}^{(i) T} K_{\Pi \Pi}^{(i)} R_{\Pi}^{(i)}, \quad \widetilde{K}_{\Pi B}=\left[R_{\Pi}^{(1) T} K_{\Pi B}^{(1)}, \ldots, R_{\Pi}^{(N) T} K_{\Pi B}^{(N)}\right],
$$

and the right-hand side $\widetilde{f}=\left[f_{B}^{T},\left(\sum_{i=1}^{N} R_{\Pi}^{(i) T} f_{\Pi}^{(i)}\right)^{T}\right]^{T}$.

In order to enforce continuity in the dual degrees of freedom, we introduce a jump operator $B_{B}=\left[B_{B}^{(1)} \ldots B_{B}^{(N)}\right]$ with $B_{B}^{(i)}$ having zero entries for the interior degrees of freedom and entries out of $\{-1,1\}$ for the dual degrees of freedom. The entries for the dual degrees of freedom are chosen such that $B_{B} u_{B}=0$ if and only if $u_{B}$ is continuous on the interface. This continuity condition is enforced by the Lagrange multipliers $\lambda$, which act between two degrees of freedom each.

The FETI-DP master system is then given by

$$
\left[\begin{array}{ccc}
K_{B B} & \widetilde{K}_{\Pi B}^{T} & B_{B}^{T} \\
\widetilde{K}_{\Pi B} & \widetilde{K}_{\Pi \Pi} & O \\
B_{B} & O & O
\end{array}\right]\left[\begin{array}{c}
u_{B} \\
\tilde{u}_{\Pi} \\
\lambda
\end{array}\right]=\left[\begin{array}{c}
f_{B} \\
\tilde{f}_{\Pi} \\
0
\end{array}\right]
$$

To solve (2.1), the variables $u_{B}$ and $\tilde{u}_{\Pi}$ are eliminated, resulting in a linear system for the Lagrange multipliers $\lambda$. By block Gaussian elimination, we thus obtain the standard FETI-DP system

$$
F \lambda=d
$$

with

$$
\begin{aligned}
& F=B_{B} K_{B B}^{-1} B_{B}^{T}+B_{B} K_{B B}^{-1} \widetilde{K}_{\Pi B}^{T} \widetilde{S}_{\Pi \Pi}^{-1} \widetilde{K}_{\Pi B} K_{B B}^{-1} B_{B}^{T} \quad \text { and } \\
& d=B_{B} K_{B B}^{-1} f_{B}+B_{B} K_{B B}^{-1} \widetilde{K}_{\Pi B}^{T} \widetilde{S}_{\Pi \Pi}^{-1}\left(\left(\sum_{i=1}^{N} R_{\Pi}^{(i) T} f_{\Pi}^{(i)}\right)-\widetilde{K}_{\Pi B} K_{B B}^{-1} f_{B}\right) .
\end{aligned}
$$

Here, the Schur complement $\widetilde{S}_{\Pi \Pi}$ for the primal variables is defined as

$$
\widetilde{S}_{\Pi \Pi}=\widetilde{K}_{\Pi \Pi}-\widetilde{K}_{\Pi B} K_{B B}^{-1} \widetilde{K}_{\Pi B}^{T} .
$$

The considered system of equations (2.2) is then solved by a Krylov subspace method, such as the (preconditioned) conjugate gradient algorithm (PCG) or GMRES (Generalized minimal residual method). In the present work, we use the PCG method and the Dirichlet preconditioner given by

$$
M_{D}^{-1}=B_{B, D}\left[\begin{array}{ll}
0 & I_{\Delta}
\end{array}\right]^{T}\left(K_{\Delta \Delta}-K_{\Delta I} K_{I I}^{-1} K_{\Delta I}^{T}\right)\left[\begin{array}{ll}
0 & I_{\Delta}
\end{array}\right] B_{B, D}^{T}=B_{D} \widetilde{S} B_{D}^{T}
$$

see $[15,16]$. Here, $I_{\Delta}$ is the identity matrix acting on the dual degrees of freedom. The matrices $B_{B, D}$ and $B_{D}$ are scaled variants of $B_{B}$ and $B$, respectively. We consider the $\rho$-scaling approach; see, e.g., [42, 63]. In this case, the scaling matrices $D^{(i)}: \operatorname{range}(B) \rightarrow \operatorname{range}(B)$, $i=1, \ldots, N$, are diagonal matrices. Note, that also non-diagonal scaling matrices exist, e.g., resulting from deluxe scaling; see $[6,40]$ and the references therein. 
2.3.3. Standard BDDC. For the description of the BDDC algorithm, we use the same sub-partitioning of the degrees of freedom into the index sets $I, \Gamma, \Pi$, and $\Delta$ as already introduced in Section 2.3.2. Here, we present the original BDDC formulation for the Schur complement system; see [11,52]. Equivalently, it is also possible to formulate the BDDC preconditioner as a preconditioner for the fully assembled system $K_{g} u=f_{g}$; see, e.g., [51]. Please note that the BDDC method is dual to the FETI-DP method, and therefore the condition number bounds for both methods are closely related; see [50,53] and Section 2.3.4.

In contrast to the FETI-DP method, we now use a slightly different ordering of the variables to describe the BDDC method. In particular, for this section, we introduce the block diagonal matrices

$$
\begin{aligned}
& K_{\Pi \Pi}=\operatorname{diag}\left(K_{\Pi \Pi}^{(1)}, \ldots, K_{\Pi \Pi}^{(N)}\right), \quad K_{\Pi I}=\operatorname{diag}\left(K_{\Pi I}^{(1)}, \ldots, K_{\Pi I}^{(N)}\right), \quad \text { and } \\
& K_{\Pi \Delta}=\operatorname{diag}\left(K_{\Pi \Delta}^{(1)}, \ldots, K_{\Pi \Delta}^{(N)}\right)
\end{aligned}
$$

as well as the corresponding right-hand sides

$$
\begin{aligned}
f_{I}^{T} & :=\left(f_{I}^{(1) T}, \ldots, f_{I}^{(N) T}\right), \quad f_{\Delta}^{T}:=\left(f_{\Delta}^{(1) T}, \ldots, f_{\Delta}^{(N) T}\right), \quad \text { and } \\
f_{\Pi}^{T} & :=\left(f_{\Pi}^{(1) T}, \ldots, f_{\Pi}^{(N) T}\right) .
\end{aligned}
$$

The matrices $K_{I I}, K_{I \Delta}, K_{\Delta I}, R_{\Delta}$, and $R_{\Pi}$ are defined analogously to Section 2.3.2. Thus, the global block matrix $K_{\mathrm{BDDC}}$ for the BDDC algorithm can be written as

$$
K_{\mathrm{BDDC}}=\left[\begin{array}{lll}
K_{I I} & K_{I \Delta} & K_{I \Pi} \\
K_{\Delta I} & K_{\Delta \Delta} & K_{\Delta \Pi} \\
K_{\Pi I} & K_{\Pi \Delta} & K_{\Pi \Pi}
\end{array}\right] .
$$

Here, we use the sub-index 'BDDC' to distinguish the matrices in this section from the global matrices used in FETI-DP (see Section 2.3.2). Please note, that $K_{\mathrm{BDDC}}$ is assembled only inside the subdomains and not across the interface. In fact, $K_{\mathrm{BDDC}}$ can be obtained from $K$ defined in Section 2.3.2 by row and column permutations. The global elimination of the inner variables $u_{I}$ yields the unassembled Schur complement

$$
S_{\mathrm{BDDC}}=\left[\begin{array}{ll}
S_{\Delta \Delta} & S_{\Delta \Pi} \\
S_{\Pi \Delta} & S_{\Pi \Pi}
\end{array}\right]=\left[\begin{array}{ll}
K_{\Delta \Delta} & K_{\Delta \Pi} \\
K_{\Pi \Delta} & K_{\Pi \Pi}
\end{array}\right]-\left[\begin{array}{c}
K_{\Delta I} \\
K_{\Pi I}
\end{array}\right] K_{I I}^{-1}\left[\begin{array}{ll}
K_{I \Delta} & K_{I \Pi}
\end{array}\right]
$$

as well as the corresponding right-hand side

$$
g_{\mathrm{BDDC}}=\left[\begin{array}{c}
g_{\Delta} \\
g_{\Pi}
\end{array}\right]=\left[\begin{array}{l}
f_{\Delta}-K_{\Delta I} K_{I I}^{-1} f_{I} \\
f_{\Pi}-K_{\Pi I} K_{I I}^{-1} f_{I}
\end{array}\right] .
$$

In the BDDC algorithm, we use a dual assembly operator $R_{\Delta}^{T}=\left(R_{\Delta}^{(1) T}, \ldots, R_{\Delta}^{(N) T}\right)$ instead of the Boolean jump operator from FETI-DP to enforce continuity in the dual variables. The unpreconditioned BDDC system then corresponds to the global primal Schur complement system $S_{g} u_{g}=g_{g}$ with the assembled global Schur complement given as

$$
S_{g}=\left[\begin{array}{cc}
R_{\Delta}^{T} & 0 \\
0 & R_{\Pi}^{T}
\end{array}\right]\left[\begin{array}{ll}
S_{\Delta \Delta} & S_{\Delta \Pi} \\
S_{\Pi \Delta} & S_{\Pi \Pi}
\end{array}\right]\left[\begin{array}{cc}
R_{\Delta} & 0 \\
0 & R_{\Pi}
\end{array}\right]
$$

and

$$
g_{g}=\left[\begin{array}{cc}
R_{\Delta}^{T} & 0 \\
0 & R_{\Pi}^{T}
\end{array}\right] g_{\mathrm{BDDC}}
$$




\section{ETNA}

Kent State University and

Johann Radon Institute (RICAM)

As for the FETI-DP algorithm, we use a preconditioner to accelerate the convergence of the iterative solver. In the present work, we again use the preconditioned conjugate gradient algorithm and the Dirichlet preconditioner given by

$$
M_{D, \mathrm{BDDC}}^{-1}=\left[\begin{array}{cc}
R_{\Delta, D}^{T} & 0 \\
0 & I_{\Pi}
\end{array}\right] \widetilde{S}_{\mathrm{BDDC}}^{-1}\left[\begin{array}{cc}
R_{\Delta, D} & 0 \\
0 & I_{\Pi}
\end{array}\right]
$$

Here, $\widetilde{S}_{\mathrm{BDDC}}$ denotes the primally assembled Schur complement matrix and $R_{\Delta, D}$ a scaled variant of the dual assembly operator $R_{\Delta}$.

2.3.4. Condition number bounds. Let us briefly recall the classic condition number bounds for both the FETI-DP and the BDDC methods. In two dimensions, the FETI-DP method with a standard vertex coarse space satisfies the polylogarithmic condition number bound

$$
\kappa\left(M_{D}^{-1} F\right) \leq C\left(1+\log \left(\frac{H}{h}\right)\right)^{2}
$$

with $C$ independent of $H$ and $h$; see [45, 46, 48]. However, this condition number bound does only hold under certain assumptions, e.g., for constant or slowly varying coefficients within each subdomain; see, e.g., [63]. In three dimensions, the preconditioned FETI-DP method with a standard vertex coarse space performs less well and cannot retain the condition number bound (2.3). Therefore, enforcing additional coarse constraints based on averages over edges or faces was proposed by several authors; see, e.g., [16, 46, 48]. Then, the condition number bound (2.3) also holds in three dimensions for heterogeneous coefficients that are constant within each subdomain or for slowly varying coefficients; see, e.g., [48]. In [42, Section 7], weighted edge averages for coefficient jumps not aligned with the interface were studied numerically for the FETI-DP algorithm. In this article, we propose a different approach to enhance the coarse space using generalized weighted edge or face averages, which is strongly motivated by the adaptive coarse space in Section 2.3.6. Please note that in [53] it was shown that the BDDC and the FETI-DP methods have, except for some eigenvalues equal to 0 and 1 , the same spectra (see also [50] for an alternative proof). Thus, all the above mentioned condition number estimates for FETI-DP are also valid for the BDDC algorithm.

2.3.5. Enforcement of additional coarse constraints. As mentioned in the introduction as well as in Section 2.3.4, using exclusively vertex constraints to enhance the coarse space is often not sufficient to obtain a robust algorithm if highly complex coefficient functions are used. In general, different approaches to implement coarse space enrichments for FETIDP and BDDC exist. Common approaches are deflation or balancing approaches [40, 44] and a transformation-of-basis approach $[43,46]$. In the present paper, the deflation and balancing approaches are only applied to the FETI-DP method since using deflation for the BDDC method is not equivalent to the BDDC using a transformation of basis; see [44]. Thus, we use a generalized transformation-of-basis approach to enhance additional coarse constraints for the BDDC method; see [33]. Explicitly transformed local stiffness matrices can become dense when using face constraints in three dimensions. To obtain an efficient parallel implementation, we use instead the equivalent approach of local saddle point problems as suggested in [47, Section 4.2.2].

2.3.6. An adaptive FETI-DP and BDDC method. Since the classic condition number bound (2.3) is only valid under certain restrictive assumptions concerning the coefficient function or the material distribution, several adaptive coarse space techniques have been developed to overcome this limitation $[31,32,33,39,40,54,55,57,58]$. The basic idea of 
most of these methods is to use additional coarse modes or primal constraints obtained by solving localized eigenvalue problems on edges, local interfaces, or subdomains to enhance the coarse space. Our new frugal constraints, proposed here, are strongly motivated by an adaptive approach which has successfully been applied to FETI-DP and BDDC for various heterogeneous model problems $[31,54,55]$. For completeness, let us first give a short description of this adaptive approach.

Let $\mathcal{X}_{i j} \subset \partial \Omega_{i} \cap \partial \Omega_{j}$, e.g., $\mathcal{X}_{i j}$ could be a face $F_{i j}$ or an edge $E_{i j}$. Then, for $\mathcal{X}_{i j}$ between two neighboring subdomains $\Omega_{i}$ and $\Omega_{j}$, a single eigenvalue problem has to be solved. We first introduce the restriction of the jump matrix $B$ to an equivalence class $\mathcal{X}_{i j}$. Let $B_{\mathcal{X}_{i j}}=\left(B_{\mathcal{X}_{i j}}^{(i)}, B_{\mathcal{X}_{i j}}^{(j)}\right)$ be the submatrix of $\left(B^{(i)}, B^{(j)}\right)$ with the rows consisting of exactly one 1 and one -1 and being zero elsewhere. By $B_{D, \mathcal{X}_{i j}}=\left(B_{D, \mathcal{X}_{i j}}^{(i)}, B_{D, \mathcal{X}_{i j}}^{(j)}\right)$ we denote the corresponding scaled jump matrix defined by taking the same rows of $\left(B_{D}^{(i)}, B_{D}^{(j)}\right)$. Let $S_{i j}=\operatorname{diag}\left(S^{(i)}, S^{(j)}\right)$ with $S^{(i)}$ and $S^{(j)}$ being the Schur complements of $K^{(i)}$ and $K^{(j)}$, respectively, with respect to the interface variables. We further define $P_{D_{i j}}=B_{D, \mathcal{X}_{i j}}^{T} B_{\mathcal{X}_{i j}}$ as a local version of the jump operator $P_{D}=B_{D}^{T} B$. Then, according to [31, 54], one has to solve the generalized eigenvalue problem:

$$
\left\langle P_{D_{i j}} v_{i j}, S_{i j} P_{D_{i j}} w_{i j}\right\rangle=\mu_{i j}\left\langle v_{i j}, S_{i j} w_{i j}\right\rangle \quad \forall v_{i j} \in\left(\operatorname{ker} \mathrm{S}_{\mathrm{ij}}\right)^{\perp} .
$$

For an explicit expression of the positive definite right-hand side operator on the subspace $\left(k e r \mathrm{~S}_{\mathrm{ij}}\right)^{\perp}$, two orthogonal projection matrices $\Pi_{i j}$ and $\bar{\Pi}_{i j}$ are used; see, e.g., [40]. One would then select all eigenvectors $w_{i j}^{l}, l=1, \ldots, L$, belonging to the eigenvalues $\mu_{i j}^{l}$, $l=1, \ldots, L$, that are larger than a user-defined tolerance $T O L$, and enforce the constraints $B_{D, \mathcal{X}_{i j}} S_{i j} P_{D_{i j}} w_{i j}^{l}, l=1, \ldots, L$, in each iteration, e.g., with a projector preconditioning or a transformation-of-basis approach. By enhancing the FETI-DP and BDDC coarse spaces with these constraints, we obtain the condition number bound

$$
\kappa\left(\widetilde{M}^{-1} F\right) \leq \widetilde{C} \cdot T O L,
$$

with $\widetilde{C}$ independent of $H$ and $h$; see [31, 40,54]. In particular, the constant $\widetilde{C}$ only depends on geometric constants of the domain decomposition, i.e., on the maximum number of edges of a subdomain in two dimensions or on the maximum number of faces of a subdomain and the maximum multiplicity of an edge in three dimensions, but is independent of the contrast of the coefficient. As already mentioned, the set-up and the solution of the eigenvalue problems take up a significant amount of time in a parallel implementation. Here, we aim to approximate the respective constraints resulting from the first eigenmodes by constructing generalized weighted averages along certain equivalence classes $\mathcal{X}_{i j}$. As for the construction of the aforementioned adaptive coarse space, we also apply the operators $B_{D, \mathcal{X}_{i j}} S_{i j} P_{D_{i j}}$ to our computed weighted averages on each edge or face.

3. A frugal coarse space. Our new frugal (FR) coarse space is strongly motivated by the adaptive approach described in Section 2.3.6. However, in contrast to adaptive coarse spaces, our new constraints do not require the solution of any eigenvalue problems or the explicit computation of Schur complements and are thus computationally very cheap. Instead, we aim to compute a low-dimensional approximation of the adaptive coarse space. Furthermore, the new frugal coarse space can be interpreted as a generalization of the weighted edge averages suggested in [42, Section 7, p. 1412]; cf. Section 3.4. It can be combined with arbitrary FETI-DP and BDDC scalings, e.g., $\rho$-scaling [42] or deluxe-scaling [6], and is robust for a broader range of heterogeneities, as shown by the numerical experiments in Section 4 and 

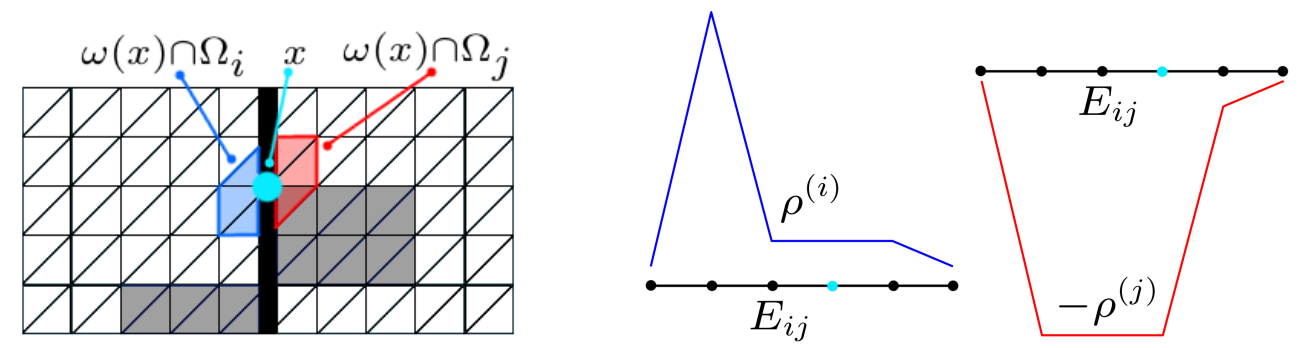

FIG. 3.1. Left: Visualization of the construction of an edge constraint in $2 D$ for a given heterogeneous coefficient distribution. Middle: Maximum coefficient per finite element node of $E_{i j}$ with respect to $\Omega_{i}$. Right: Maximum coefficient per finite element node of $E_{i j}$ with respect to $\Omega_{j}$.

Section 5. Please note that first results for diffusion problems in two dimensions using the new edge constraints instead of constraints resulting from the solution of a specific edge eigenvalue problem were already published in $[27,28]$.

3.1. Motivation and construction in two dimensions. Our new approach is strongly motivated by the generalized eigenvalue problem (2.4) from the adaptive coarse space [31, 54], which can equivalently be written as

$$
\left\langle\mathcal{H}\left(P_{D_{i j}} v_{i j}\right), K_{i j} \mathcal{H}\left(P_{D_{i j}} w_{i j}\right)\right\rangle=\mu_{i j}\left\langle\mathcal{H}\left(v_{i j}\right), K_{i j} \mathcal{H}\left(w_{i j}\right)\right\rangle=\mu_{i j}\left\langle\mathcal{H}\left(v_{E_{i j}}\right), K_{i j} \mathcal{H}\left(w_{E_{i j}}\right)\right\rangle,
$$

where $K_{i j}=\operatorname{diag}\left(K^{(i)}, K^{(j)}\right)$ and $\mathcal{H}(\cdot)$ is the discrete harmonic extension from the interface of $\Omega_{i}$ and $\Omega_{j}$ to the interior of the subdomains $\Omega_{i}$ and $\Omega_{j}$; cf., e.g., [63, Section 4]. Therefore, as described in Section 2.3.6, all eigenmodes with

$$
\mu_{i j}=\frac{\left|\mathcal{H}\left(P_{D_{i j}} v_{i j}\right)\right|_{K_{i j}}}{\left|\mathcal{H}\left(v_{i j}\right)\right|_{K_{i j}}}>T O L
$$

are selected and then used to construct the adaptive constraints. In particular, this corresponds to the case where $\left|\mathcal{H}\left(P_{D_{i j}} v_{i j}\right)\right|_{K_{i j}}$ is large, i.e., of the order of the contrast of the coefficient function, while $\left|\mathcal{H}\left(v_{i j}\right)\right|_{K_{i j}}$ is small.

Now, in our new frugal approach, we propose a specific construction of an edge function $v_{E_{i j}}$ which often has the desired properties of the energies $\left|\mathcal{H}\left(P_{D_{i j}} v_{E_{i j}}\right)\right|_{K_{i j}}$ and $\left|\mathcal{H}\left(v_{E_{i j}}\right)\right|_{K_{i j}}$. Therefore, the space spanned by all edge functions $v_{E_{i j}}$ can be regarded as a lower-dimensional approximation of the original adaptive coarse space.

Let us first consider the case of diffusion in two dimensions. In this case, we only construct constraints corresponding to the edges of the domain decomposition. We denote by $E_{i j}$ the edge between two neighboring subdomains $\Omega_{i}$ and $\Omega_{j}$, and by $\omega(x)$ the support of the finite element basis functions associated with a finite element node $x \in\left(\Omega_{i} \cup \Omega_{j}\right)$. For each $x$ on $\partial \Omega_{i}$ or $\partial \Omega_{j}$, respectively, we compute

$$
\widehat{\rho}^{(i)}(x)=\max _{y \in \omega(x) \cap \Omega_{i}} \rho(y) \quad \text { and } \quad \hat{\rho}^{(j)}(x)=\max _{y \in \omega(x) \cap \Omega_{j}} \rho(y) .
$$

Now, we define $v_{E_{i j}}^{(l)}$ on $\partial \Omega_{l}$, for $l=i, j$, by

$$
v_{E_{i j}}^{(l)}(x):= \begin{cases}\widehat{\rho}^{(l)}(x) & x \in \partial \Omega_{l} \backslash \Pi^{(l)}, \\ 0 & x \in \Pi^{(l)}\end{cases}
$$

and $v_{E_{i j}}^{T}:=\left(v_{E_{i j}}^{(i) T},-v_{E_{i j}}^{(j) T}\right)$. Here, $\Pi^{(l)}$ denotes the index set of all local primal variables. See also Figure 3.1 for a visualization of this function. As can be observed for example in 


\section{ETNA}

Kent State University and

Johann Radon Institute (RICAM)
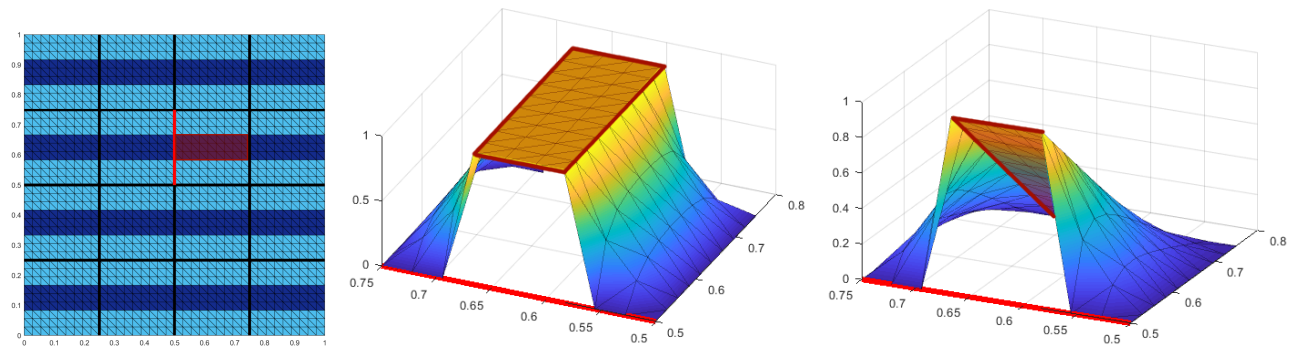

FIG. 3.2. Visualization of the new constraints for a concrete coefficient distribution. Left: Coefficient distribution with one channel associated with a large coefficient crossing each subdomain. Dark blue corresponds to the large coefficient (1e6) and light blue to the small coefficient (1). Visualization for $4 \times 4$ subdomains and $H / h=9$. Middle: Visualization of the discrete harmonic extension $\mathcal{H}\left(v_{E_{i j}}\right)$ for one floating subdomain. The visualized constraint leads to an energy of 17.49. Right: Visualization of the discrete harmonic extension $\mathcal{H}\left(P_{D_{i j}} v_{E_{i j}}\right)$ for the same floating subdomain. The visualized constraint leads to an energy of $6.67 \mathrm{e}+5$.

Figure 3.2 (middle) and Figure 3.3 (middle), the energy $\left|\mathcal{H}\left(v_{E_{i j}}\right)\right|_{K_{i j}}$ is low. On the other hand, the energy $\left|\mathcal{H}\left(P_{D_{i j}} v_{E_{i j}}\right)\right|_{K_{i j}}$ is large for those two examples; see Figure 3.2 (right), where the energy is large due to the homogeneous Dirichlet boundary enforced by $P_{D_{i j}}$, and Figure 3.3 (right), where the energy is large due to the gradient of the scaled jump $P_{D_{i j}} v_{E_{i j}}$ on the edge.

We obtain the edge constraint by $c_{E_{i j}}:=B_{D_{i j}} S_{i j} P_{D_{i j}} v_{E_{i j}}$ as in the adaptive coarse space; cf. Section 2.3.6. We denote by $B_{D_{i j}}$ the submatrix of $\left(B_{D}^{(i)}, B_{D}^{(j)}\right)$ with the rows restricted to the edge $E_{i j}$. We further define $S_{i j}=\operatorname{diag}\left(S^{(i)}, S^{(j)}\right)$, where $S^{(i)}$ and $S^{(j)}$ are the Schur complement matrices of $K^{(i)}$ and $K^{(j)}$, respectively, with respect to the interface variables, as well as the operator $P_{D_{i j}}=B_{D_{i j}}^{T} B_{i j}$.

The construction (3.2) can be further simplified by exploiting the fact that the scaled jump operator $P_{D_{i j}}$ is zero everywhere except on $E_{i j}$. Therefore, our new constraint is constructed instead as

$$
v_{E_{i j}}^{(l)}(x)= \begin{cases}\hat{\rho}^{(l)}(x) & x \in E_{i j}, \\ 0 & x \in \partial \Omega_{l} \backslash E_{i j},\end{cases}
$$

for $l=i, j$; cf. the definition in [27]. In particular, due to the subsequent application of $P_{D}$, both definitions of $v_{E_{i j}}^{(l)}$ result in the same constraints $c_{E_{i j}}$.

For our parallel implementation, we use the latter definition of $v_{E_{i j}}^{(l)}$. There, we exploit the extension by zero to the remaining interface $\partial \Omega_{l} \backslash E_{i j}$ and reduce the application of several $P_{D_{i j}}$ to a few global applications of $P_{D}$; see also Section 5 for more details.

3.2. Diffusion in three dimensions. The respective case of diffusion problems in three dimensions is relatively analogous to the case of diffusion problems in two dimensions in Section 3.1, with the main difference that we now compute our new constraint for faces $F_{i j}$ or, alternatively, closed faces $\bar{F}_{i j}$ between neighboring subdomains $\Omega_{i}$ and $\Omega_{j}$ instead of edges $E_{i j}$. Let us first define

$$
v_{F_{i j}}^{(l)}(x)= \begin{cases}\widehat{\rho}^{(l)}(x) & x \in F_{i j} \\ 0 & x \in \partial \Omega_{l} \backslash F_{i j}\end{cases}
$$

and

$$
v_{\bar{F}_{i j}}^{(l)}(x)= \begin{cases}\hat{\rho}^{(l)}(x) & x \in \bar{F}_{i j}, \\ 0 & x \in \partial \Omega_{l} \backslash \bar{F}_{i j} .\end{cases}
$$



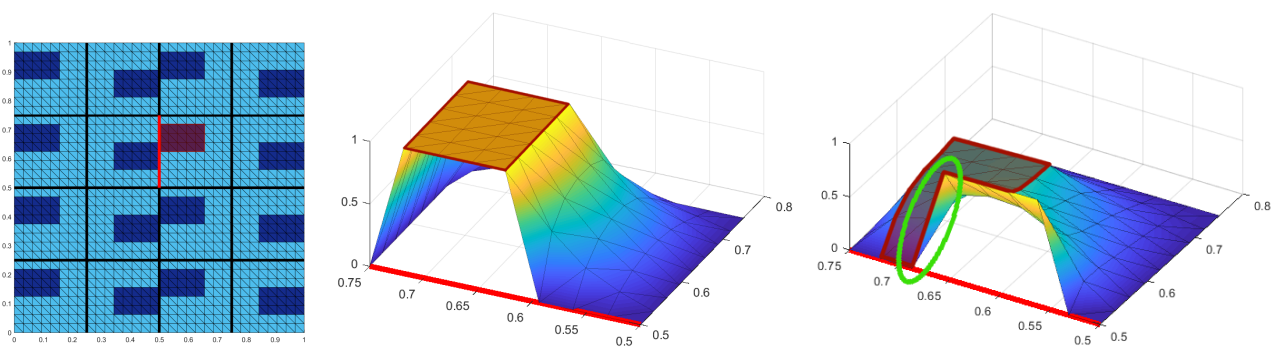

FIG. 3.3. Visualization of the new constraints for a concrete coefficient distribution. Left: Coefficient distribution with boxes associated with a large coefficient. Dark blue corresponds to the large coefficient $(1 \mathrm{e}+6)$ and light blue to the small coefficient (1). Visualization for $4 \times 4$ subdomains and $H / h=8$. Middle: Visualization of the discrete harmonic extension $\mathcal{H}\left(v_{E_{i j}}\right)$ for one floating subdomain, which is nearly constant in the area with a large coefficient marked in red. The visualized constraint leads to an energy of 17.39. Right: Visualization of the discrete harmonic extension $\mathcal{H}\left(P_{D_{i j}} v_{E_{i j}}\right)$ for the same floating subdomain, which has large gradients (see green ellipse) in the area with a large coefficient marked in red. The visualized constraint leads to an energy of $1.96 \mathrm{e}+6$.

Analogously to the two-dimensional case, we obtain our constraints $c_{\bar{F}_{i j}}$ by applying the operator $B_{D_{i j}} S_{i j} P_{D_{i j}}$ to either $v_{F_{i j}}$ or $v_{\bar{F}_{i j}}$, respectively. Let us remark that, due to the structure of the Schur complement matrix $S_{i j}$ as well as $P_{D_{i j}}$, in both cases $c_{\bar{F}_{i j}}$ may have nonzero entries on the closed face, i.e., on the open face $F_{i j}$ and on all edges $E_{m}$, $m=1,2, \ldots, M$, belonging to the closed face $\bar{F}_{i j}$. We can therefore split a constraint $c_{\bar{F}_{i j}}$ into a constraint $c_{F_{i j}}$ on the open face and constraints $c_{E_{m}}, m=1, \ldots, M$, on the neighboring edges. The same approach of splitting the constraints into face- and edge-related parts is proposed in [31].

Depending on whether we also include the edge-related parts into the coarse space or not, four different possible variants exist of how we concretely enhance the coarse space related to faces:

FR1: Construct $v \bar{F}_{i j}$ for each closed face $\bar{F}_{i j}$, and enforce both the edge-related parts $c_{E_{m}}$, $m=1, \ldots, M$, as well as the part related to the open face of $c_{F_{i j}}$.

FR2: Construct $v \frac{T}{F_{i j}}$ for each closed face $\bar{F}_{i j}$, but just extract the terms of $c_{F_{i j}}$ related to the open face, while discarding the respective edge-related parts.

FR3: Construct $v_{F_{i j}}^{T}$ for each open face $F_{i j}$, and enforce both the edge-related parts $c_{E_{m}}$, $m=1, \ldots, M$, as well as the part related to the open face of $c_{F_{i j}}$.

FR4: Construct $v_{F_{i j}}^{T}$ for each open face $F_{i j}$, but just extract the terms of $c_{F_{i j}}$ related to the open face, while discarding the respective edge-related parts.

We will compare the robustness of the four different variants in terms of condition number estimates and iteration counts in the numerical experiments in Section 4. Let us remark that FR1 implements the complete constraints which are built analogously to the two-dimensional case. With respect to a parallel implementation, FR4 is the most promising since the different open faces have no intersections with each other and therefore many local operations, such as, e.g., applications of $P_{D_{i j}}$, can be grouped to global operations and can be carried out for all faces simultaneously; see Section 5.

3.3. Linear elasticity in three dimensions. For the case of three-dimensional linear elasticity problems, we know that, when applying the FETI-DP or BDDC algorithms, we need six constraints, i.e., six rigid body modes, to control the null spaces for subdomains which have boundaries that do not intersect $\partial \Omega$. For a generic domain $\widehat{\Omega}$ with diameter $H$, the basis 
for the null space $\operatorname{ker}(\varepsilon)$ is given by the three translations

$$
r_{1}:=\left[\begin{array}{l}
1 \\
0 \\
0
\end{array}\right], \quad r_{2}:=\left[\begin{array}{l}
0 \\
1 \\
0
\end{array}\right], \quad r_{3}:=\left[\begin{array}{l}
0 \\
0 \\
1
\end{array}\right]
$$

and the three linear rotations

$$
r_{4}:=\frac{1}{H}\left[\begin{array}{c}
x_{2}-\widehat{x}_{2} \\
-x_{1}+\widehat{x}_{1} \\
0
\end{array}\right], \quad r_{5}:=\frac{1}{H}\left[\begin{array}{c}
-x_{3}+\widehat{x}_{3} \\
0 \\
x_{1}-\widehat{x}_{1}
\end{array}\right], \quad r_{6}:=\frac{1}{H}\left[\begin{array}{c}
0 \\
x_{3}-\widehat{x}_{3} \\
-x_{2}+\widehat{x}_{2}
\end{array}\right]
$$

where $\widehat{x} \in \widehat{\Omega}$ is the center of the linear rotations; see, e.g., [42, Section 2]. We now construct six weighted constraints per face to obtain a robust coarse space. Basically, these constraints are based on the maximum coefficients per element, i.e., the maximum Young modulus $E>0$, as well as on the three translations and the three rotations for the respective face between two neighboring subdomains. Let us describe the concrete construction of the coarse constraints in some more detail. Let $F_{i j}$ be the open face between two neighboring subdomains $\Omega_{i}$ and $\Omega_{j}$, respectively, and $\bar{F}_{i j}$ the closed face. For each finite element node $x$ on $\partial \Omega_{i}$ or $\partial \Omega_{j}$, we compute $\widehat{E}^{(l)}(x)=\max _{y \in \omega(x) \cap \Omega_{l}} E(y)$, for $l=i, j$. Furthermore, we compute six scaled rigid body modes denoted by $\widehat{r}_{m}^{(l)}, m=1, \ldots, 6, l=i, j$, by pointwise scaling the rigid body modes $r_{m}, m=1, \ldots, 6$, with the respective vectors of the maximum coefficients $\widehat{E}^{(l)}(x)$, $l=i, j$. Note that all three degrees of freedom belonging to a given node $x \in \partial \Omega_{i} \cup \partial \Omega_{j}$ are scaled with the same value of $\widehat{E}^{(l)}(x)$, for $l=i, j$. For $m=1, \ldots, 6$ and $l=i, j$, we then define

$$
v_{F_{i j}}^{(m, l)}(x)= \begin{cases}\widehat{r}_{m}^{(l)}(x) & x \in F_{i j}, \\ 0 & x \in \partial \Omega_{l} \backslash F_{i j}\end{cases}
$$

Combining the vectors for both subdomains to $v_{F_{i j}}^{(m) T}=\left(v_{F_{i j}}^{(m, i) T},-v_{F_{i j}}^{(m, j) T}\right)$, we obtain the weights for the six face constraints as

$$
c_{\bar{F}_{i j}}^{(m)}:=B_{D_{i j}} S_{i j} P_{D_{i j}} v_{F_{i j}}^{(m)}, \quad m=1, \ldots, 6 .
$$

The variants for closed faced are defined analogously; see also (3.3). Thus, the four different variants FR1 to FR4 of coarse spaces can be implemented as in the diffusion case. Please note that the resulting constraints can, in certain cases, be linearly dependent and thus result in less than six constraints per face. Therefore, we always apply a modified Gram-Schmidt algorithm after constructing the six aforementioned constraints in our implementation. For the case of linear elasticity in two dimensions, the computation of $c_{F_{i j}}^{(m)}, m=1, \ldots, 3$, is completely analogous to the three-dimensional case. For two dimensions, we just scale all two degrees of freedom per node for a given node $x$ with the same value of $\widehat{E}^{(l)}(x)$, for $l=i, j$. Since the three-dimensional case is more general, we here chose to describe the three-dimensional case in more detail.

Due to the possible existence of hinge modes for two neighboring subdomains in case of linear elasticity problems, using only face constraints and edge constraints arising as a byproduct in the construction of the face constraints, as, e.g., in the variants FR1 and FR3, might not always lead to a robust algorithm for complex coefficient distributions. In particular, in some cases the use of additional edge constraints is necessary to obtain moderate condition number bounds as well as scalabilty. We will consider such a coefficient distribution 
in Table 4.7, where the exclusive use of face constraints is not sufficient. For this special case, we enforce additional weighted edge constraints besides the weighted face and edge constraints already introduced. The construction of our weighted edge constraints is in principle completely analogous to the aforementioned face constraints. More precisely, the construction is basically the same except that we now operate on the index set of open edges between two neighboring subdomains that do not share a face (alternatively, we can additionally construct weighted edge constraints for all edges, for simplicity, and finally apply a modified Gram-Schmidt algorithm to eliminate linearly dependent constraints). We thus obtain an additional variant, which we denote by FR5. Let us remark that we first use FR1 for all faces and ensure that we have no redundancies in the face-related and additional weighted edge constraints by applying a Gram-Schmidt orthogonalization.

3.4. Classic weighted average constraints in three dimensions. For comparison, we also consider the classic coarse spaces introduced in [42], which we briefly describe in this section. We introduce weighted averages

$$
\frac{\sum_{x_{i} \in \mathcal{X}} \hat{r}_{j}\left(x_{i}\right) u\left(x_{i}\right)}{\sum_{x_{i} \in \mathcal{X}} \hat{r}_{j}\left(x_{i}\right)^{2}}, \quad j=1, \ldots, l,
$$

on parts of the interface $\mathcal{X}$, e.g., the edges $\mathcal{E}$ or faces $\mathcal{F}$. Here, we have $l=1$ for the scalar diffusion case, and $l=3$ or $l=6$ in the case of linear elasticity. Let us remark that in the latter case only five of the six constraints might be linear independent on straight edges; see [42]. For the scalar case, we consider the weights

$$
\hat{\rho}(x)=\max _{y \in \omega(x)} \rho(y),
$$

and for the case of linear elasticity we choose

$$
\hat{E}(x)=\max _{y \in \omega(x)} E(y) .
$$

We further define pointwise

$$
\hat{r}_{1}(x)=\hat{\rho}(x)
$$

in the scalar case and

$$
\hat{r}_{j}(x)=\hat{E}(x) r_{j}(x), \quad j=1, \ldots, 6,
$$

in case of linear elasticity, where $r_{1}, r_{2}$, and $r_{3}$ are the three translations and, respectively, $r_{4}, r_{5}$, and $r_{6}$ the three rotations; see also Section 3.3. Let us remark that in [42] only weighted translations, i.e., $\hat{r}_{j}, k=1, \ldots, 3$, have been used, and thus the coarse space described in this section is in fact an extension of the robust coarse space used in [42].

4. Numerical results. In this section, we present numerical results obtained using our serial MATLAB implementations of the FETI-DP and BDDC algorithms. We consider three-dimensional stationary diffusion and linear elasticity problems on the unit cube, $\Omega=[0,1]^{3}$, with Dirichlet boundary conditions on the left-hand side of the boundary $\partial \Omega$, i.e, $\partial \Omega_{D}=0 \times[0,1]^{2}$. In all our numerical experiments, we use the $\rho$-scaling approach and, as an iterative solver, the preconditioned conjugate gradient (PCG) algorithm. As stopping criterion for PCG, we use a relative reduction of the preconditioned residual by a factor of 1e-8. As already mentioned in Section 2.3.4, we obtain the same quantitative condition number 
bounds for FETI-DP and BDDC since the two methods are dual to each other; see also [50, 53]. Therefore, we do not provide results for both methods for all tested coefficient distributions. We consider beams with large coefficients inside subdomains and with varying offsets between the subdomains as well as inclusions of large coefficients within the subdomains.

Let us remark that in addition to the considered face- or edge-based constraints, we always choose all vertices to be primal. In all tables and figures we use the following notation to distinguish between the different classic coarse spaces based on weighted averages:

e: Using vertex constraints and edge constraints (e), i.e., enforcing (3.5) for all edges $\mathcal{E}$. In case of linear elasticity, only weighted translations are enforced, i.e., $l=3$ in (3.5).

f: Using vertex constraints and face constraints (f), i.e., enforcing (3.5) for all faces $\mathcal{F}$. In case of linear elasticity, only weighted translations are enforced, i.e., $l=3$ in (3.5).

$\mathbf{f}+\mathbf{r}$ : Using vertex constraints and face constraints (f), i.e., enforcing (3.5) for all faces $\mathcal{F}$. In case of linear elasticity, translations and rotations (r) are enforced, i.e., $l=6$ in (3.5).

Let us remark that instead of fixing the global coefficient distribution, we always keep the coefficient distribution fixed for each subdomain in our weak scaling studies. Consequently, the global coefficient distribution actually changes while increasing the number of subdomains.

4.1. Variations of one beam per subdomain. We provide numerical results for straight and shifted beams with large coefficients as shown in Figure 4.1 and Figure 4.2. We consider both diffusion problems as well as linear elasticity problems and compare the results for our new frugal coarse space to the classic approach from [42]. For the straight channels that only cut through faces, in Table 4.1, all four variants FR1 to FR4 show a more or less equivalent performance. Here, the classic approach also provides comparable and robust condition number bounds and iteration counts. For the shifted channels, see Table 4.2, the edge-related variants FR1 and FR3 show a slightly better performance compared to FR2 and FR4. This effect is mostly noticeable for the linear elasticity problems presented in Table 4.2. However, also the exclusively face-based variants FR2 and FR4 show robust condition numbers in all computations. In particular, in this case, the classic weighted averages are not sufficient to provide robust algorithms. This shows that our new approach is indeed more general than classic averages and provides robustness for more complex coefficient distributions. As two additional sets of experiments, we further provide results for varying contrasts of the coefficients. In Table 4.4 we show the condition number estimates and iteration numbers for a stationary diffusion problem and the shifted beams as in Figure 4.2 with an increasing contrast between the two coefficients. As we can observe from Table 4.4, the classic coarse space performs clearly poorer than the frugal coarse space for all tested coefficient contrasts. In particular, for the larger coefficient $\rho$ being equal to $1 \mathrm{e} 4,1 \mathrm{e} 5,1 \mathrm{e} 6$, the classic coarse space clearly fails to provide a robust algorithm since the condition number estimates increase in proportion to the contrast of the coefficients. However, the frugal coarse spaces are seen to be robust with respect to the coefficient contrast. We further provide experiments for stationary diffusion problems and two straight beams of a large coefficient crossing each subdomain in Table 4.5.

As a proof that our weighted constraints work equally well for the FETI-DP and the BDDC algorithm, we further provide numerical results for the shifted channels and the BDDC algorithm in Table 4.3.

4.2. Inclusions within subdomains. Let us consider the case of inclusions of large coefficients within subdomains; see Figure 4.3. We note that we only consider the case of inclusions which have a nonempty intersection with the respective subdomain boundary. 

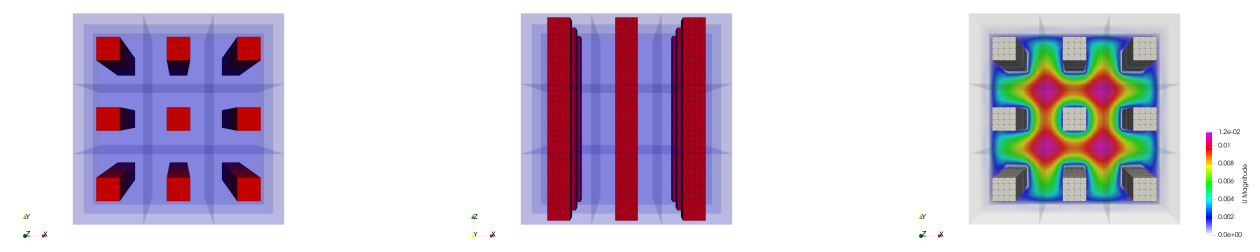

FIG. 4.1. Left and Middle: Coefficient function with one central beam per subdomain. Large coefficients are shown in red, and subdomains are shown in purple and by half-transparent slices. Right: Visualization of the corresponding solution for the stationary diffusion problem. Visualization for $3 \times 3 \times 3=27$ subdomains and $H / h=12$.
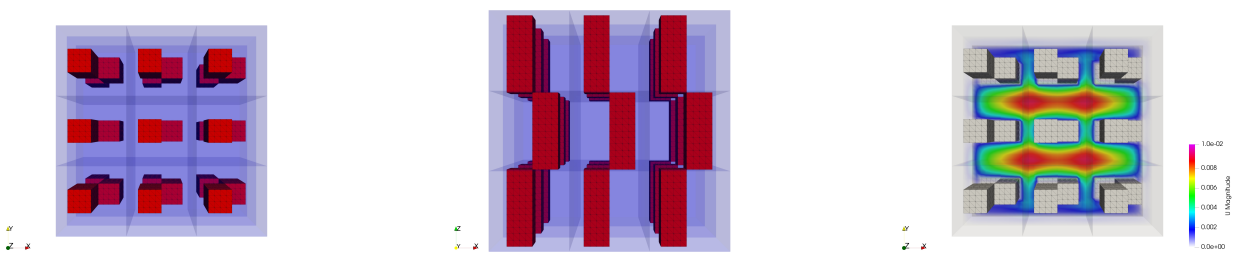

FIG. 4.2. Left and Middle: Coefficient function with one beam per subdomain with offsets. Large coefficients are shown in red, and subdomains are shown in purple and by half-transparent slices. Right: Visualization of the corresponding solution for the stationary diffusion problem. Visualization for $3 \times 3 \times 3=27$ subdomains and $H / h=12$.

For inclusions within subdomains which have a positive distance to the boundary of the respective subdomain, already standard FETI-DP and BDDC coarse spaces yield robust domain decomposition methods, and no additional constraints have to be added to the coarse spaces; see, e.g., [20] for FETI-DP or [59] for FETI. For the first set of experiments, we partition each subdomain into eight cubes of equal size and set a large coefficient in two of these cubes, which intersect only at a single vertex; see Figure 4.3. As the results in Table 4.6 show, our new face constraints lead to robust algorithms for the diffusion problem for all variants FR1 to FR4. For elasticity problems, however, the resulting algorithms including only face constraints exhibits poor convergence behaviour or even divergence; see Table 4.7 (column 1). As a comparison, we further include results for an adaptive FETI-DP method in Table 4.7. Here, we use the adaptive coarse space as proposed by Mandel and Sousedík [54] and a variant thereof as implemented by Klawonn, Kühn, and Rheinbach [31]. Basically, in these methods the solutions of certain local generalized eigenvalue problems on faces or edges are used to enrich the coarse space. We refer to $[31,54]$ for more details on this adaptive method. In Table 4.7, we denote by:

- adaptive, face EVP: the adaptive FETI-DP method from [54] using exclusively eigenvalue problems on faces;

- adaptive, edge EVP: the adaptive FETI-DP method from [31] using additional eigenvalue problems on edges to enrich the coarse space.

As the results in Table 4.7 show, the adaptive FETI-DP algorithm also displays poor convergence behavior for this specific linear elasticity problem when using exclusively face constraints. However, the use of additional edge constraints again leads to a robust algorithm, as the last column in Table 4.7 shows. This indicates that for this specific coefficient 
TABLE 4.1

Condition numbers (cond) and iteration numbers (it) for the FETI-DP algorithm for stationary diffusion and linear elasticity problems in $3 D$ with $H / h=6$ for one straight beam per subdomain as in Figure 4.1. The larger coefficient is $\rho=1 \mathrm{e} 6$ for stationary diffusion and $E=1 \mathrm{e} 6$ for linear elasticity (with $\nu=0.3$ everywhere).

\begin{tabular}{|c|c|c|c|c|c|c|c|c|c|c|}
\hline \multirow[b]{2}{*}{$\mathbf{N}$} & \multicolumn{8}{|c|}{ new approach } & \multirow{2}{*}{\multicolumn{2}{|c|}{$\begin{array}{c}\text { classic } \\
\mathbf{f}\end{array}$}} \\
\hline & \multicolumn{2}{|c|}{ FR1 } & \multicolumn{2}{|c|}{ FR2 } & \multicolumn{2}{|c|}{ FR3 } & \multicolumn{2}{|c|}{ FR4 } & & \\
\hline \multicolumn{11}{|c|}{ stationary diffusion } \\
\hline & cond & it & cond & it & cond & it & cond & it & cond & it \\
\hline $2^{3}$ & 1.25 & 5 & 1.44 & 6 & 1.25 & 5 & 1.44 & 6 & 1.44 & 7 \\
\hline $3^{3}$ & 1.25 & 6 & 1.51 & 8 & 1.25 & 6 & 1.51 & 8 & 1.51 & 10 \\
\hline $4^{3}$ & 1.25 & 6 & 1.53 & 8 & 1.25 & 6 & 1.53 & 8 & 1.53 & 10 \\
\hline \multicolumn{11}{|c|}{ linear elasticity } \\
\hline & cond & it & cond & it & cond & it & cond & it & cond & it \\
\hline $2^{3}$ & 1.59 & 10 & 2.70 & 13 & 1.59 & 10 & 2.71 & 14 & 2.71 & 14 \\
\hline $3^{3}$ & 1.63 & 11 & 2.78 & 16 & 1.62 & 10 & 2.78 & 16 & 2.78 & 15 \\
\hline $4^{3}$ & 1.63 & 11 & 2.86 & 16 & 1.62 & 10 & 2.85 & 16 & 2.85 & 16 \\
\hline
\end{tabular}

TABLE 4.2

Condition numbers (cond) and iteration numbers (it) for the FETI-DP algorithm for stationary diffusion and linear elasticity problems in $3 D$ with $H / h=6$ for one beam per subdomain with offsets as in Figure 4.2. The larger coefficient is $\rho=1 \mathrm{e} 6$ for stationary diffusion and $E=1 \mathrm{e} 6$ for linear elasticity (with $\nu=0.3$ everywhere).

\begin{tabular}{|c|c|c|c|c|c|c|c|c|c|c|}
\hline \multirow[b]{2}{*}{$\mathbf{N}$} & \multicolumn{8}{|c|}{ new approach } & \multirow{2}{*}{\multicolumn{2}{|c|}{$\begin{array}{c}\text { classic } \\
\mathbf{f}\end{array}$}} \\
\hline & \multicolumn{2}{|c|}{ FR1 } & \multicolumn{2}{|c|}{ FR2 } & \multicolumn{2}{|c|}{ FR3 } & \multicolumn{2}{|c|}{ FR4 } & & \\
\hline \multicolumn{11}{|c|}{ stationary diffusion } \\
\hline & cond & it & cond & it & cond & it & cond & it & cond & it \\
\hline $2^{3}$ & 1.36 & 8 & 1.72 & 10 & 1.36 & 8 & 1.68 & 10 & 43613.4 & 16 \\
\hline $3^{3}$ & 1.41 & 9 & 1.88 & 11 & 1.41 & 9 & 1.83 & 11 & 46336.5 & 47 \\
\hline $4^{3}$ & 1.41 & 9 & 1.91 & 12 & 1.41 & 9 & 1.86 & 12 & 46622.0 & 78 \\
\hline \multicolumn{11}{|c|}{ linear elasticity } \\
\hline & cond & it & cond & it & cond & it & cond & it & cond & it \\
\hline $2^{3}$ & 1.92 & 12 & 3.92 & 18 & 1.90 & 13 & 3.68 & 17 & 37930.9 & 54 \\
\hline $3^{3}$ & 1.90 & 12 & 4.48 & 19 & 1.89 & 12 & 4.37 & 19 & 68238.5 & 124 \\
\hline $4^{3}$ & 1.92 & 12 & 4.91 & 21 & 1.90 & 12 & 4.76 & 21 & 76027.6 & 264 \\
\hline
\end{tabular}

distribution with two cubes of large coefficients per subdomain only intersecting at a single vertex, a hinge mode exists in the case of linear elasticity, which is not controlled by our new face constraints. Thus, we also consider the variant of enforcing our new weighted edge constraints in addition to the already constructed face average constraints, denoted by FR5; see Section 3.3. In the second column of Table 4.7, we present numerical results concerning this variant, given the inclusions per subdomain intersecting only in a single vertex as before. Please note that the additional use of explicit edge constraints in principle corresponds to the solution of explicit edge eigenvalue problems for subdomains that do not share a face in the context of the adaptive coarse space proposed in [31]. To obtain a robust algorithm, we again use a modified Gram-Schmidt algorithm to eliminate all linearly dependent constraints resulting from edge-related parts of weighted face constraints and the explicitly constructed edge constraints.

4.3. Reducing the coarse space dimension. As already stated above, our proposed coarse space is of a similar size as the classic coarse space introduced in [42]. In particular, we 
TABLE 4.3

Condition numbers (cond) and iteration numbers (it) for the BDDC algorithm for stationary diffusion and linear elasticity problems in $3 D$ with $H / h=6$ for one beam per subdomain with offsets as in Figure 4.2. The larger coefficient is $\rho=1 \mathrm{e} 6$ for stationary diffusion and $E=1 \mathrm{e} 6$ for linear elasticity (with $\nu=0.3$ everywhere).

\begin{tabular}{|c|c|c|c|c|c|c|c|c|}
\hline $\mathbf{N}$ & \multicolumn{2}{|c|}{ FR1 } & \multicolumn{2}{|c|}{ FR2 } & \multicolumn{2}{|c|}{ FR3 } & \multicolumn{2}{|c|}{ FR4 } \\
\hline \multicolumn{9}{|c|}{ stationary diffusion } \\
\hline & cond & it & cond & it & cond & it & cond & it \\
\hline $2^{3}$ & 1.29 & 8 & 1.72 & 11 & 1.28 & 8 & 1.68 & 11 \\
\hline $3^{3}$ & 1.35 & 9 & 1.88 & 12 & 1.33 & 9 & 1.83 & 11 \\
\hline $4^{3}$ & 1.35 & 9 & 1.90 & 13 & 1.34 & 9 & 1.86 & 12 \\
\hline \multicolumn{9}{|c|}{ linear elasticity } \\
\hline & cond & it & cond & it & cond & it & cond & it \\
\hline $2^{3}$ & 1.89 & 12 & 3.91 & 18 & 1.88 & 12 & 3.90 & 17 \\
\hline $3^{3}$ & 1.87 & 11 & 4.45 & 19 & 1.86 & 11 & 4.37 & 19 \\
\hline $4^{3}$ & 1.88 & 11 & 4.92 & 20 & 1.88 & 12 & 4.76 & 20 \\
\hline
\end{tabular}

TABLE 4.4

Condition numbers (cond) and iteration numbers (it) for the FETI-DP algorithm for stationary diffusion problems in $3 D$ with $H / h=9$ for one beam per subdomain with offsets as in Figure 4.2. The larger coefficient is varied as coeff- $2=10^{e}, e \in\{0,3,4,5,6\}$, and the smaller coefficient is 1 .

\begin{tabular}{|c|c|c|c|c|c|c|c|}
\hline $\mathbf{N}$ & coeff-2 & \multicolumn{3}{|c|}{ frugal, FR1 } & \multicolumn{3}{|c|}{ classic, $\mathbf{f}$} \\
\hline \multicolumn{8}{|c|}{ stationary diffusion } \\
\hline & & $\mathrm{H} / \mathrm{h}$ & cond & it & $\mathrm{H} / \mathrm{h}$ & cond & it \\
\hline $4^{3}$ & $1 \mathrm{e} 0$ & 9 & 1.60 & 10 & 9 & 2.13 & 13 \\
\hline $4^{3}$ & $1 \mathrm{e} 3$ & 9 & 1.63 & 10 & 9 & 53.41 & 38 \\
\hline $4^{3}$ & $1 \mathrm{e} 4$ & 9 & 1.64 & 10 & 9 & 525.91 & 59 \\
\hline $4^{3}$ & $1 \mathrm{e} 5$ & 9 & 1.65 & 10 & 9 & 5250.98 & 78 \\
\hline $4^{3}$ & $1 \mathrm{e} 6$ & 9 & 1.93 & 12 & 9 & 52501.63 & 87 \\
\hline
\end{tabular}

construct FR constraints over all faces and/or edges. However, for many real world problems, i.e., problems with realistic coefficient distributions, constraints on certain faces or edges are not necessary and can be omitted. Therefore, we further propose a modified variant to reduce the size of the coarse space, which only requires moderate additional effort.

Since our constraints are inspired from the eigenvalue problems introduced in $[54,55]$, we can use the quotient (3.1) corresponding to the eigenvalue problems to estimate the constraints that are actually required for a robust algorithm. Numerical results have shown that for faces or edges, for which the left-hand side of the specific eigenvalue problem yields a high energy and the respective right-hand side yields a low energy, additional coarse constraints are essential for robustness. For the convenience of the reader, we explicitly write down the right-hand side $(R S)$ and the left-hand side $(L S)$ of the eigenvalue problem (2.4):

$$
L S:=P_{D_{i j}}^{T} S_{i j} P_{D_{i j}} \quad \text { and } \quad R S:=S_{i j}
$$

See $[54,55,60]$ for more technical details on the eigenvalue problem. To estimate the energy of our new constraints, we evaluate the product terms $R S e:=v_{F_{i j}}^{T} R S v_{F_{i j}}$ and $L S e:=v_{F_{i j}}^{T} L S v_{F_{i j}}$ in three dimensions, or $R S e:=v_{E_{i j}}^{T} R S v_{E_{i j}}$ and $L S e:=v_{E_{i j}}^{T} L S v_{E_{i j}}$ in two dimensions, respectively. We further evaluate the ratio $L S e / R S e$ for all faces (and, depending on the chosen variant, for all edges). For the reduced dimensional variant, we now only integrate those face constraints into the coarse space for which the ratio $L S e / R S e$ is 
TABLE 4.5

Condition numbers (cond) and iteration numbers (it) for the FETI-DP algorithm for stationary diffusion problems in $3 D$ with $H / h=10$ for two straight beams per subdomain. The larger coefficient is varied as coeff- $2=10^{e}, e \in\{0,3,4,5,6\}$, and the smaller coefficient is 1 .

\begin{tabular}{ll|lrl|lrl} 
N & coeff-2 & \multicolumn{3}{c|}{ frugal, FR1 } & \multicolumn{3}{c}{ classic, f } \\
\hline \hline \multicolumn{9}{c}{ stationary diffusion } \\
\hline & & H/h & cond & it & H/h & cond & it \\
\hline $4^{3}$ & $1 \mathrm{e} 0$ & 10 & 1.64 & 10 & 10 & 2.23 & 13 \\
$4^{3}$ & $1 \mathrm{e} 3$ & 10 & 5.88 & 20 & 10 & 6.18 & 21 \\
$4^{3}$ & $1 \mathrm{e} 4$ & 10 & 51.08 & 40 & 10 & 52.51 & 41 \\
$4^{3}$ & $1 \mathrm{e} 5$ & 10 & 503.30 & 64 & 10 & 517.20 & 67 \\
$4^{3}$ & $1 \mathrm{e} 6$ & 10 & 5025.51 & 86 & 10 & 5164.14 & 92
\end{tabular}

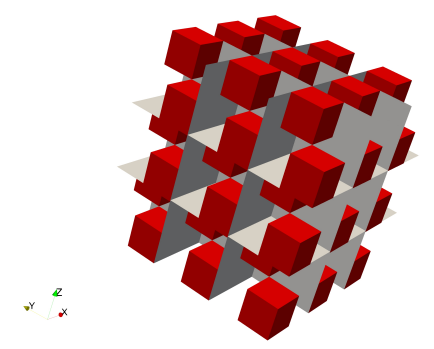

FIG. 4.3. Coefficient distribution with inclusions of large coefficients within subdomains. Large coefficients are shown in red, and subdomains are shown by grey slices. Visualization for $3 \times 3 \times 3$ subdomains and $H / h=12$.

above a user-defined tolerance $T O L$; see, e.g., [26] for a discussion on the choice of $T O L$. We denote this reduced coarse space variant by FR2 red.. We show first numerical results concerning this reduced variant for straight channels in Table 4.8. For all shown cases, we are able to reduce the dimension of the coarse space to exactly one third of the original size while preserving both robust condition numbers and iteration counts. In Table 4.9, we further show numerical results for five spherical inclusions of different radii as depicted in Figure 5.5; see also Section 5 for detailed parallel results concerning this specific coefficient distribution. For the diffusion case, we are able to reduce the size of the coarse space up to a factor of 2.4. For the case of linear elasticity, the coarse space dimension is reduced by a factor of up to 1.6.

5. Parallel numerical results. We have added the new coarse space FR4 to our parallel BDDC implementation described in [36] and compare it with classic coarse spaces based on weighted edge or face translations and rotations as introduced in [42]; see also Section 3.4. We again consider stationary diffusion and linear elasticity problems. Unless stated otherwise, Dirichlet boundary conditions on the complete boundary are used. As in Section 4, we use the preconditioned conjugate gradient (PCG) algorithm as the iterative solver for our computations. As the stopping criterion for PCG we again use a relative reduction of the preconditioned residual by a factor of $1 \mathrm{e}-8$.

5.1. Parallel implementation and computational effort. Let us remark that our PETScbased parallel implementation presented in [36] is based on a BDDC preconditioner for the complete system $K_{g}$. Therefore, no Schur complement systems are built explicitly. Conse- 
TABLE 4.6

Condition numbers (cond) and iteration numbers (it) for the FETI-DP algorithm for diffusion problems in $3 D$ with $H / h=8$ with two inclusions per subdomain as in Figure 4.3. The larger coefficient is $\rho_{1}=1 \mathrm{e} 6$, and the smaller coefficient is $\rho_{2}=1$.

\begin{tabular}{c|cc|cc|c|c|cc} 
N & FR1 & \multicolumn{2}{c|}{ FR2 } & \multicolumn{2}{c|}{ FR3 } & \multicolumn{2}{c}{ FR4 } \\
\hline \hline \multicolumn{8}{c}{ stationary diffusion } \\
\hline & cond & it & cond & it & cond & it & cond & it \\
\hline $2^{3}$ & 3.55 & 14 & 3.55 & 14 & 3.55 & 14 & 3.55 & 14 \\
$3^{3}$ & 4.05 & 20 & 4.05 & 20 & 4.05 & 20 & 4.05 & 20 \\
$4^{3}$ & 4.41 & 22 & 4.41 & 22 & 4.41 & 21 & 4.41 & 22
\end{tabular}

TABLE 4.7

Condition numbers (cond), iteration numbers (it), and the size of the coarse space (\# c.) for the FETI-DP algorithm for linear elasticity problems in $3 D$ with $H / h=6$ with two inclusions per subdomain as in Figure 4.3 . The larger coefficient is $E_{1}=1 \mathrm{e} 6$, and the smaller coefficient is $E_{2}=1$, with $\nu=0.3$ everywhere.

\begin{tabular}{|c|c|c|c|c|c|c|c|c|c|c|c|c|}
\hline \multirow[b]{2}{*}{$\mathbf{N}$} & \multicolumn{6}{|c|}{ new approach } & \multicolumn{6}{|c|}{ adaptive approach } \\
\hline & \multicolumn{3}{|c|}{ FR1 } & \multicolumn{3}{|c|}{ FR5 } & \multicolumn{3}{|c|}{ face EVP } & \multicolumn{3}{|c|}{ edge EVP } \\
\hline \multicolumn{13}{|c|}{ linear elasticity } \\
\hline & \# c. & cond & it & \# c. & cond & it & \# c. & cond & it & \# c. & cond & it \\
\hline $2^{3}$ & 288 & 25158 & 54 & 324 & 1.72 & 10 & 39 & 58679 & 58 & 173 & 3.70 & 15 \\
\hline $3^{3}$ & 1452 & 18530 & 180 & 1668 & 1.73 & 10 & 164 & 87156 & 246 & 838 & 3.42 & 20 \\
\hline $4^{3}$ & 4032 & 19626 & 232 & 4680 & 1.74 & 10 & 429 & 114882 & 471 & 2319 & 3.44 & 20 \\
\hline
\end{tabular}

quently, we also avoid the computation of the matrix $S_{i j}$, which is used for the construction of our edge or face constraints; see (3.4). Instead of computing $S^{(i)} w_{\Gamma}^{(i)}$, we always equivalently compute

$$
R_{\Gamma}^{(i)} \cdot K^{(i)} \cdot\left[\begin{array}{c}
-\left(K_{I I}^{(i)}\right)_{I}^{-1} K_{\Gamma I}^{(i) T} \\
I
\end{array}\right] w_{\Gamma}^{(i)}
$$

where $R_{\Gamma}^{(i)}$ is the restriction from the complete subdomain $\Omega_{i}$ to its interface and the operator $-\left(K_{I I}^{(i)}\right)^{-1} K_{\Gamma I}^{(i) T} w_{\Gamma}^{(i)}$ is the discrete harmonic extension of $w_{\Gamma}^{(i)}$ from the interface to the interior of $\Omega_{i}$. For the parallel implementation, we chose FR4 out of the different variants, since the coarse space can be computed cheaply with less effort than a few CG iterations; see the discussion below. Additionally, FR4 showed promising results for most problems considered in the previous section.

Parallel computation of FR4. For simplicity, we describe the implementation for the scalar diffusion case. Considering linear elasticity problems, the building blocks are identical and just called six times for each of the six rigid body modes.

In our BDDC implementation, we do not enforce the constraints $c_{F_{i j}}=B_{D_{i j}} S_{i j} P_{D_{i j}} v_{F_{i j}}$ directly in the space of interface jumps, e.g., by a projector preconditioning approach, but equivalently, the constraint $C_{F_{i j}}^{(i) T}=-C_{F_{i j}}^{(j) T}$ is enforced by a generalized transformation-ofbasis approach. Here, we consider $C_{F_{i j}}=\left(C_{F_{i j}}^{(i) T}, C_{F_{i j}}^{(j) T}\right)$ with $C_{F_{i j}}=P_{D_{i j}}^{T} S_{i j} P_{D_{i j}} v_{F_{i j}}$. With $P_{D}=I-E_{D}$, we compute $C_{F_{i j}}=\left(I-E_{D_{i j}}\right)^{T} S_{i j}\left(I-E_{D_{i j}}\right) v_{F_{i j}}$ instead, which is more convenient in the context of BDDC.

In FR4, only open faces are considered, and thus the computation of $x_{F_{i j}}:=\left(I-E_{D_{i j}}\right) v_{F_{i j}}$ can be carried out for all faces at once. Here we exploit the fact that the functions $v_{F_{i j}}$ can be 


\section{ETNA}

Kent State University and

Johann Radon Institute (RICAM)

TABLE 4.8

Condition numbers (cond) and iteration numbers (it) for the FETI-DP algorithm for linear elasticity problems in $3 D$ with $H / h=6$ for one straight beam per subdomain as in Figure 4.1. Variant with reduced coarse space dimension and $T O L=10$. The larger coefficient is $E_{1}=1 \mathrm{e} 6$, and the smaller coefficient is $E_{2}=1$, with $\nu=0.3$ everywhere.

\begin{tabular}{|c|c|c|c|c|c|c|}
\hline $\mathbf{N}$ & \multicolumn{3}{|c|}{ FR2 } & \multicolumn{3}{|c|}{ FR2 red. } \\
\hline \multicolumn{7}{|c|}{ linear elasticity } \\
\hline & \# c. & cond & it & \# c. & cond & it \\
\hline $2^{3}$ & 72 & 2.70 & 13 & 24 & 3.54 & 15 \\
\hline $3^{3}$ & 324 & 2.78 & 16 & 108 & 4.17 & 19 \\
\hline $4^{3}$ & 864 & 2.86 & 16 & 288 & 4.44 & 20 \\
\hline
\end{tabular}

TABLE 4.9

Condition numbers (cond) and iteration numbers (it) for the FETI-DP algorithm for diffusion and linear elasticity problems in $3 D$ with $H / h=10$ for five spherical inclusions as in Figure 5.5. Variant with reduced coarse space dimension and TOL $=100$. The larger coefficient is $\rho=1 \mathrm{e} 6$ for stationary diffusion and $E=1 \mathrm{e} 6$ for linear elasticity (with $\nu=0.3$ everywhere).

\begin{tabular}{|c|c|c|c|c|c|c|}
\hline $\mathbf{N}$ & \multicolumn{3}{|c|}{ FR2 } & \multicolumn{3}{|c|}{ FR2 red. } \\
\hline \multicolumn{7}{|c|}{ stationary diffusion } \\
\hline & \# c. & cond & it & \# c. & cond & it \\
\hline $2^{3}$ & 12 & 6.90 & 19 & 5 & 17.02 & 22 \\
\hline $3^{3}$ & 54 & 3.64 & 17 & 29 & 18.92 & 27 \\
\hline $4^{3}$ & 144 & 5.59 & 22 & 74 & 18.55 & 39 \\
\hline \multicolumn{7}{|c|}{ linear elasticity } \\
\hline & \# c. & cond & it & \# c. & cond & it \\
\hline $2^{3}$ & 72 & 31.89 & 44 & 55 & 61.83 & 54 \\
\hline $3^{3}$ & 324 & 70.35 & 46 & 199 & 70.36 & 64 \\
\hline $4^{3}$ & 864 & 232.36 & 67 & 633 & 430.11 & 95 \\
\hline
\end{tabular}

chosen to be zero on the remaining interface; see Section 3.1 and following arguments. Therefore, all values $v_{F_{i j}}$ for all faces are collected into a single vector $v$. The remaining interface components in $v$ can be set arbitrarily. Then, $x:=\left(I-E_{D}\right) v$ can be computed in parallel using the parallel implementation of $E_{D}$ based on PETSc VecScatter operations; see [36] for details on the implementation. All $x_{F_{i j}}$ for all open faces can be easily obtained from $x$ by extracting local values on the faces and extending them by zero to the remaining local interface. Let us remark that with $x_{F_{i j}}=\left(x_{F_{i j}}^{(i)}, x_{F_{i j}}^{(j)}\right)$ the computation of $S_{i j} x_{F_{i j}}$ actually decomposes into two local computations $S^{(l)} x_{F_{i j}}^{(l)}, l=i, j$, which are carried out following (5.1). This process is completely local, but equation (5.1) has to be computed separately for each face of a subdomain. The results for all local faces can again be collected into a single vector $y$, and again $I-E_{D}$ can be applied globally. Finally, all coarse constraints are locally extracted face by face from $\left(I-E_{D}\right) y$.

Computational effort. The FR4 coarse space is of similar size compared to classic faceor edge-based coarse spaces, but the computation of the constraints is more costly. Therefore, the effort will only pay off compared to classic approaches such as, e.g., those from [42], if a sufficient number of CG iterations is saved. Let us remark that the computation of the constraints from FR4 is cheap compared to the computation of any adaptive constraints which requires the solution of local generalized eigenvalue problems. To obtain a useful estimate for the computational effort, we compare the cost to compute the coarse constraints to the cost of some CG iterations, i.e., some applications of the system matrix and the BDDC preconditioner. 
In a single application of the BDDC preconditioner, $\left(I-E_{D}\right)$ is applied twice as here in the construction of the coarse space FR4. The discrete harmonic extension which appears in (5.1) is also applied twice in each application of the BDDC preconditioner. Finally, in each CG iteration, the matrix $K^{(i)}$ is applied once to a vector in the application of the system matrix. Considering six faces per subdomain for a regular decomposition, the construction of the coarse space thus costs less than six CG iterations. Assuming that the computation of the discrete harmonic extensions is the most expensive operation in this process, we can approximately compare the cost with the cost of three CG iterations. Therefore, if we can save at least three CG iterations, FR4 will pay off. Of course, since the computation of the constraints of FR4 does not include any coarse solve, it will be even less expensive, especially for problems with many subdomains and compute cores. Therefore, the estimate with $3 \mathrm{CG}$ iterations is in fact way too pessimistic.

For the case of linear elasticity with six rigid body modes for each of the six faces, we end up with an approximate cost of 18 CG iterations-following the argumentation above. Again, we expect much less effort especially for larger problems. In practice, e.g., considering the example from Figure 5.4, we measure a time of approximately 8.1 CG iterations to construct the coarse space for the 48 subdomain case and only $2.4 \mathrm{CG}$ iterations for the 3072 subdomain case. Let us finally remark that the construction might be more expensive for irregular domain decompositions with more than six faces per subdomain.

5.2. Sanity check with a checkerboard problem. As a sanity check of our software, we provide results for the classic checkerboard problem shown in Figure 5.1; see Figure 5.2 for the results. As expected, using $\rho$-scaling, the coarse space with vertices and edges performs slightly better compared to face-based approaches since an acceptable edge path can be found; see [42]. Additionally, FR4 and the classic face constraints defined in Section 3.4 deliver similar results.

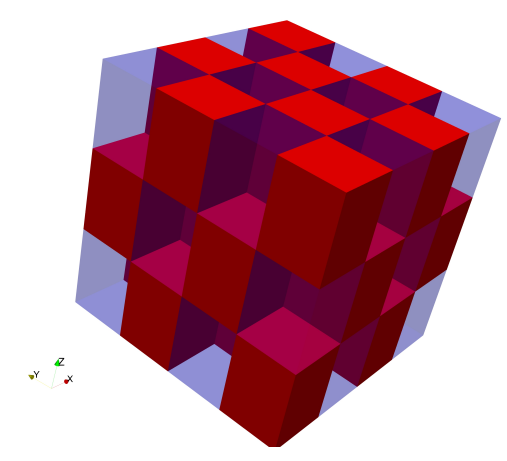

FIG. 5.1. Coefficient distribution in a checkerboard pattern with constant coefficients per subdomain. Large coefficients are shown in red. Visualization for $4 \times 4 \times 3$ subdomains and $H / h=12$.

5.3. Straight and shifted channels. We provide parallel weak scaling results for straight and shifted channels as depicted in Figure 4.1 and Figure 4.2. We consider both a diffusion problem (see Figure 5.3) as well as a linear elasticity problem (see Figure 5.4). For all examples, face constraints are necessary, and while for the straight channels FR4 is more or less equally robust as the classic face constraints, it is superior for the model problem with shifted channels and up to a factor 9.2 faster in time-to-solution for the diffusion problem. For the case of linear elasticity, the time-to-solution is reduced by a factor of up to 2.5 . 


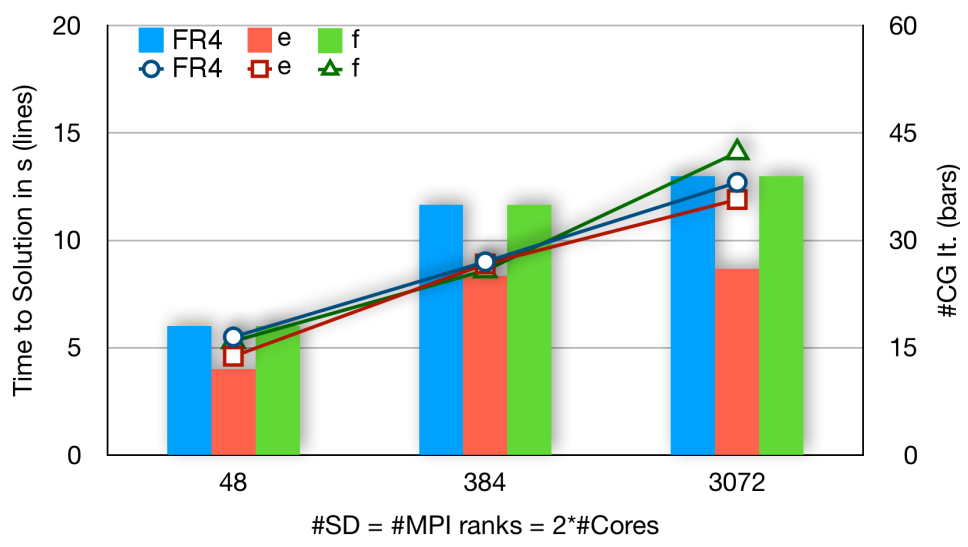

FIG. 5.2. Parallel weak scaling test; stationary diffusion problem with a constant coefficient on each subdomains, varying in a checkerboard pattern. The larger coefficient is 1e6, and the smaller coefficient is 1.
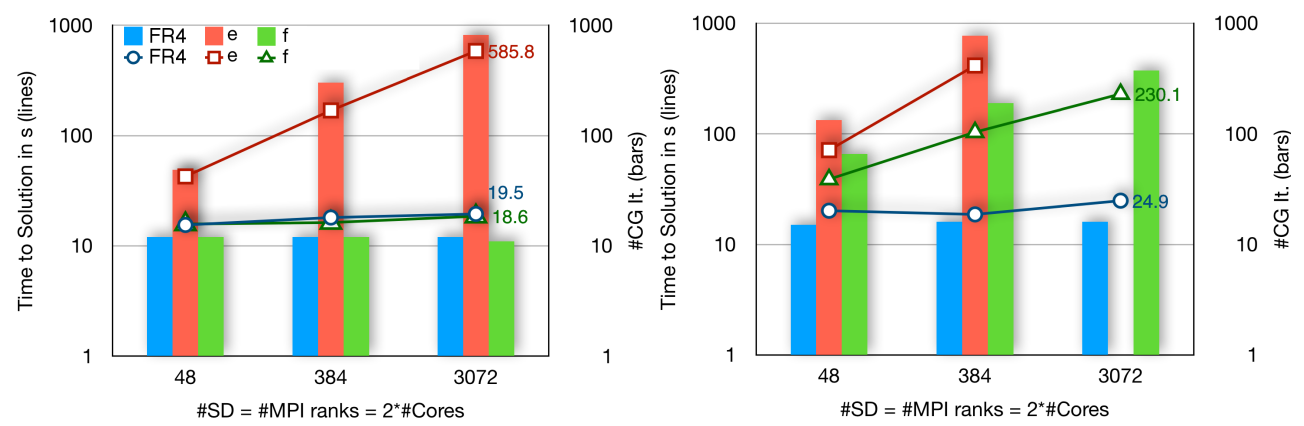

FIG. 5.3. Parallel weak scaling test; stationary diffusion problem with a single channel crossing each subdomain; larger coefficient $\rho=1 \mathrm{e} 6$ inside channels and $\rho=1$ in the remaining domain. Left: Straight channels. Right: Shifted channels. Missing data corresponds to runs which did not converge within 1000 CG iterations.

5.4. Five spherical inclusions and an RVE. To investigate more general and more realistic coefficient distributions that are chosen independently of the domain decomposition, we consider two additional examples.

Five Spherical Inclusions. First, we consider five spherical inclusions of different radii; see Figure 5.5. Let us remark that considering our structured mesh, each voxel is discretized by six tetrahedral finite elements, and these six elements always share the same coefficient. Each voxel within the five spheres will have an identical large coefficient, i.e., a large $\rho$ in the diffusion case or a large $E$ in the linear elasticity case. The remaining matrix material will have a smaller coefficient. For a decomposition into 384 regular subdomains, we depict a face between two subdomains (see Figure 5.6 (left)) and mark the parts of the spheres which lie inside these two subdomains in blue and red. Zooming in (Figure 5.6 (right)), we observe a similar situation as in the case of the shifted channels. Additionally, the spherical inclusions cut or touch also edges and vertices. Considering this model problem, FR4 outperforms all tested classic approaches significantly; see Table 5.1. Especially for the largest considered coefficient jump of 1e6, only FR4 is robust for both diffusion and linear elasticity problems.

$R V E$. Second, we consider an RVE (representative volume element) of a dual-phase steel consisting of the two material phases martensite and ferrite, representing the microscopic structure of a DP600 steel and obtained by an EBDS (electronic backscatter diffraction) 

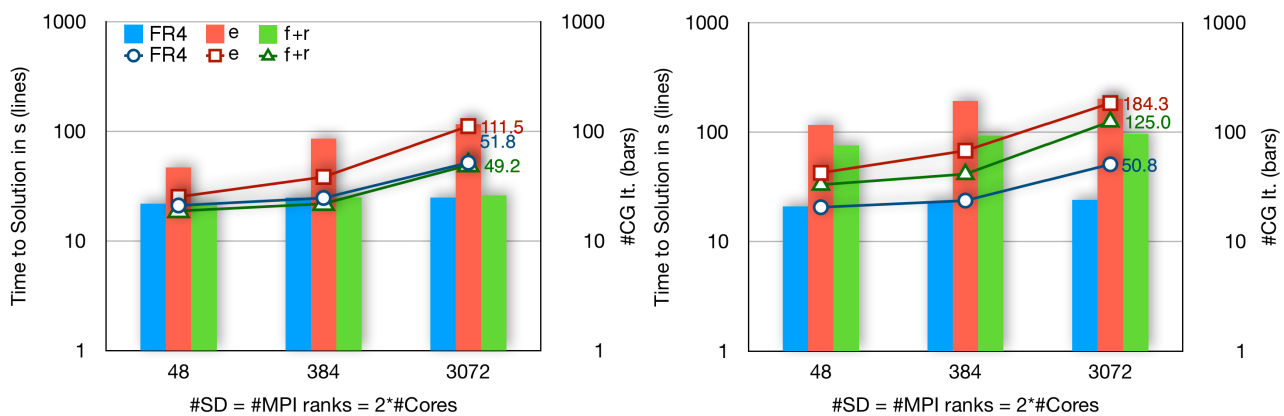

FIG. 5.4. Parallel weak scaling test; linear elasticity problem with one channel crossing each subdomain; larger coefficient $E=210000$ inside channels and $E=210$ in matrix material. Left: Straight channels. Right: Shifted channels.

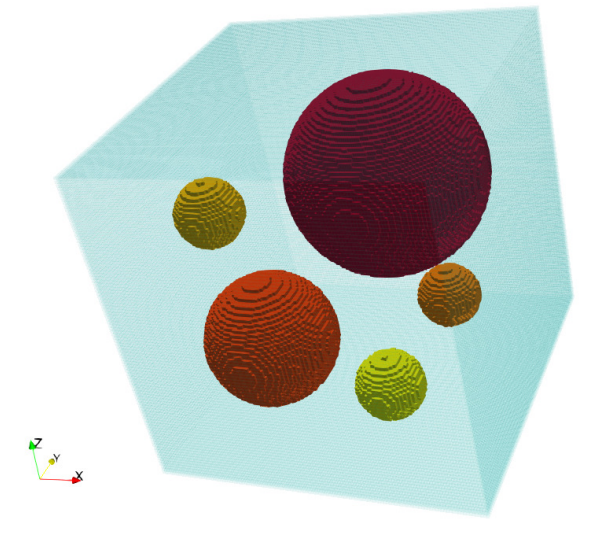

FIG. 5.5. Five spheres with different radii in the unit cube. Resolution of $128 \times 128 \times 96$ voxel corresponding to computations with $H / h=16$ and $8 \times 8 \times 6=384$ subdomains in Table 5.1.
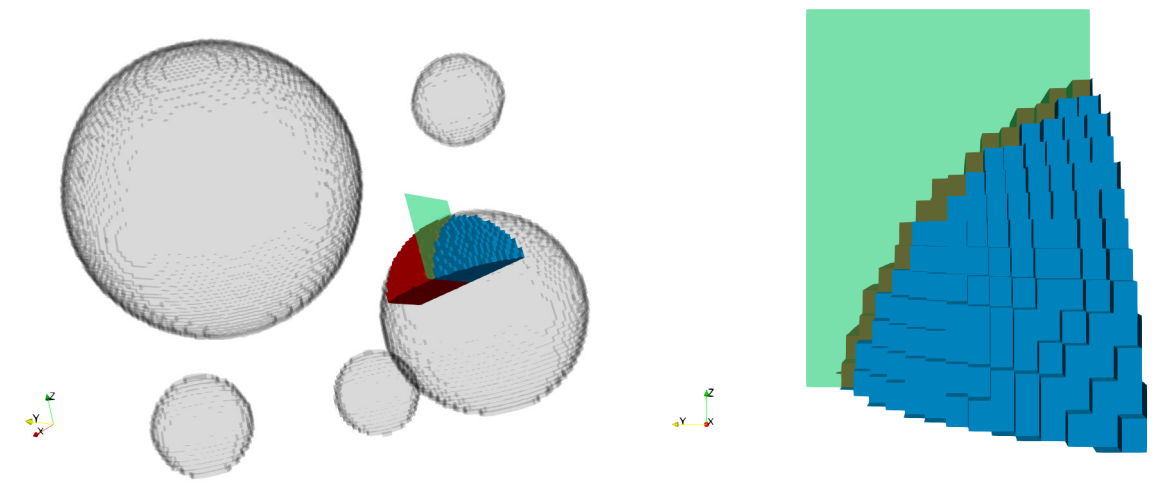

FIG. 5.6. Left: Example visualization of the coefficient function in Figure 5.5 for two neighboring subdomains, marked in red and blue, and the face between those subdomains, marked in green. Right: Zoom-in of the coefficient jump along the green face between two neighboring subdomains. 


\section{ETNA}

Kent State University and

Johann Radon Institute (RICAM)

TABLE 5.1

Coefficient distribution with five spherical inclusions of different size; see Figure 5.5. Resolution of $128 \times$ $128 \times 96 \operatorname{voxel}(H / h=16)$ or $192 \times 192 \times 144$ voxel $(H / h=24)$. Each voxel is discretized with six finite elements; stationary diffusion: coefficients of $1 \mathrm{e} 3$ or $1 \mathrm{e} 6$ inside the spheres and 1 in the matrix material; linear elasticity: coefficients of $E=210000$ or 210000000 in the spheres and $E=210$ in the matrix material; $\nu=0.3$ everywhere. Decomposition into 384 subdomains; computed on 192 cores. Using $\rho$-scaling. $\mathbf{v}$ stands for vertex constraints, e for weighted edge translations, $\mathbf{f}$ for weighted face translations, and $\mathbf{r}$ for weighted edge or face rotations; TtS abbreviates Time-to-Solution and \# c. the size of the coarse space.

\begin{tabular}{|c|c|c|c|c|c|c|c|c|}
\hline & \multicolumn{4}{|c|}{ stationary diffusion } & \multicolumn{4}{|c|}{ linear elasticity } \\
\hline \multicolumn{9}{|c|}{ coefficient jump $1 \mathrm{e}+3 ; H / h=16$} \\
\hline coarse space & \# c. & cond & it & $\mathrm{TtS}$ & \# c. & cond & it & $\mathrm{TtS}$ \\
\hline FR4 & 1237 & $7.42 \mathrm{e}+0$ & 19 & $1.7 \mathrm{~s}$ & 6687 & $3.05 \mathrm{e}+1$ & 44 & $19.9 \mathrm{~s}$ \\
\hline $\mathrm{f}$ & 1237 & $1.04 \mathrm{e}+2$ & 45 & $2.7 \mathrm{~s}$ & 3711 & $1.60 \mathrm{e}+3$ & 259 & $55.1 \mathrm{~s}$ \\
\hline$f+r$ & - & - & - & - & 6687 & $9.76 \mathrm{e}+2$ & 144 & $38.3 \mathrm{~s}$ \\
\hline $\mathrm{e}$ & 1141 & $5.00 \mathrm{e}+3$ & 135 & $7.3 \mathrm{~s}$ & 3423 & $7.00 \mathrm{e}+2$ & 212 & $48.7 \mathrm{~s}$ \\
\hline \multicolumn{9}{|c|}{ coefficient jump $1 \mathrm{e}+3 ; H / h=24$} \\
\hline coarse space & \# c. & cond & it & $\mathrm{TtS}$ & \# c. & cond & it & $\mathrm{TtS}$ \\
\hline FR4 & 1237 & $8.73 e+0$ & 22 & $8.3 \mathrm{~s}$ & 6687 & $4.77 \mathrm{e}+1$ & 54 & $100.5 \mathrm{~s}$ \\
\hline $\mathrm{f}$ & 1237 & $3.83 \mathrm{e}+1$ & 41 & $9.7 \mathrm{~s}$ & 3711 & $1.68 \mathrm{e}+3$ & 269 & $264.2 \mathrm{~s}$ \\
\hline$f+r$ & - & - & - & - & 6687 & $2.07 \mathrm{e}+2$ & 114 & $143.9 \mathrm{~s}$ \\
\hline $\mathrm{e}$ & 1141 & $1.08 \mathrm{e}+4$ & 194 & $36.1 \mathrm{~s}$ & 3423 & $9.17 \mathrm{e}+2$ & 238 & $245.9 \mathrm{~s}$ \\
\hline \multicolumn{9}{|c|}{ coefficient jump $1 \mathrm{e}+6 ; H / h=16$} \\
\hline coarse space & \# c. & cond & it & $\mathrm{TtS}$ & \# c. & cond & it & $\mathrm{TtS}$ \\
\hline FR4 & 1237 & $7.51 \mathrm{e}+0$ & 19 & $1.7 \mathrm{~s}$ & 6687 & $3.22 \mathrm{e}+1$ & 47 & $20.7 \mathrm{~s}$ \\
\hline $\mathrm{f}$ & 1237 & $1.02 \mathrm{e}+5$ & 189 & $11.3 \mathrm{~s}$ & 3711 & $1.46 \mathrm{e}+6$ & $>1000$ & $>204.8 s$ \\
\hline$f+r$ & - & - & - & - & 6687 & $5.40 \mathrm{e}+5$ & $>1000$ & $>222.0 \mathrm{~s}$ \\
\hline $\mathrm{e}$ & 1141 & $4.97 \mathrm{e}+6$ & 283 & $14.6 \mathrm{~s}$ & 3423 & $6.87 \mathrm{e}+5$ & $>1000$ & $>210.6 \mathrm{~s}$ \\
\hline \multicolumn{9}{|c|}{ coefficient jump $1 \mathrm{e}+6 ; H / h=24$} \\
\hline coarse space & \# c. & cond & it & $\mathrm{TtS}$ & \# c. & cond & it & $\mathrm{TtS}$ \\
\hline FR4 & 1237 & $8.84 \mathrm{e}+0$ & 21 & $6.7 \mathrm{~s}$ & 6687 & $5.14 \mathrm{e}+1$ & 57 & $103.1 \mathrm{~s}$ \\
\hline $\mathrm{f}$ & 1237 & $3.54 \mathrm{e}+4$ & 195 & $36.4 \mathrm{~s}$ & 3711 & $1.36 \mathrm{e}+6$ & $>1000$ & $>889.9 \mathrm{~s}$ \\
\hline$f+r$ & - & - & - & - & 6687 & $1.01 \mathrm{e}+5$ & $>1000$ & $>915.5 \mathrm{~s}$ \\
\hline $\mathrm{e}$ & 1141 & $1.07 \mathrm{e}+7$ & 434 & $78.5 \mathrm{~s}$ & 3423 & $8.78 \mathrm{e}+5$ & $>1000$ & $>900.4 \mathrm{~s}$ \\
\hline
\end{tabular}

measurement. This RVE is part of a larger structure presented in [8]. The martensitic inclusions are depicted in red in Figure 5.7 (left), and the ferritic matrix material is marked in blue. The RVE is decomposed into 512 subdomains (see Figure 5.7 (left)), and a linear elastic solution is shown in Figure 5.7 (right). We use large coefficients in the martensitic phase and small coefficients in the ferritic phase and use the coefficient distribution for diffusion and linear elasticity computations; see Table 5.2. The most realistic computation is the linear elasticity problem with a coefficient jump of $1 \mathrm{e}+3$, since these parameters are most representative for a real dual-phase steel. Let us remark that for large deformations, steel shows a plastic behavior, and therefore a linear elastic material model is not sufficient anymore. Considering Table 5.2, FR4 again shows the best performance, and the iteration counts are acceptable in all cases, even though for the linear elasticity problem the condition number is also large. Here, additional constraints are necessary e.g., adaptive constraints obtained by solving certain localized eigenvalue problems. 

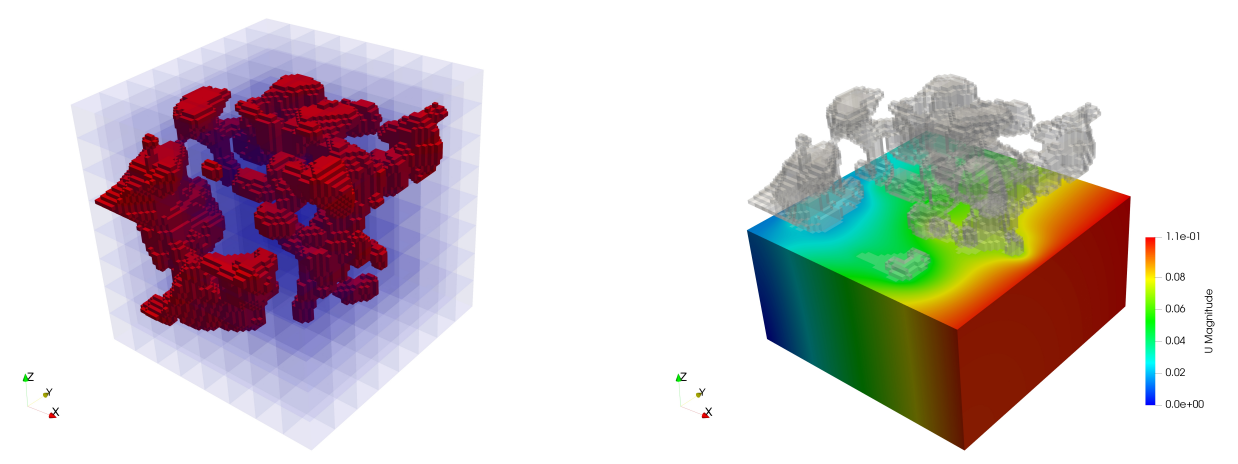

FIG. 5.7. Coefficient distribution on a representative volume element (RVE). Left: Visualization of the domain decomposition into $8 \times 8 \times 8=512$ subdomains and $H / h=16$. Large coefficients are shown in red, and subdomains are shown in purple and by half-transparent slices. Right: Visualization of the corresponding solution of the RVE. Based on data from [8].

TABLE 5.2

Coefficient distribution obtained by an EBSD measurement of a dual-phase steel; see Figure 5.7. Resolution of $192 \times 192 \times 192$ voxel $(H / h=24)$. Each voxel is discretized with six finite elements; stationary diffusion: coefficients of $1 \mathrm{e} 3$ or $1 \mathrm{e} 6$ inside the inclusions and 1 in the matrix material; linear elasticity: coefficients of $E=210000$ or $E=210000000$ in the inclusios and $E=210$ in the matrix material; $\nu=0.3$ everywhere. Decomposition into 512 subdomains; computed on 256 cores. Using $\rho$-scaling. $\mathbf{v}$ stands for vertex constraints, $\mathbf{f}$ for weighted face translations, and $\mathbf{r}$ for weighted face rotations; $\mathbf{T t S}$ abbreviates Time-to-Solution and \# $\mathbf{c}$. the size of the coarse space.

\begin{tabular}{|c|c|c|c|c|c|c|c|c|}
\hline & \multicolumn{4}{|c|}{ stationary diffusion } & \multicolumn{4}{|c|}{ linear elasticity } \\
\hline \multicolumn{9}{|c|}{ coefficient jump $1 \mathrm{e}+3 ; H / h=24$} \\
\hline coarse space & \# c. & cond & it & $\mathrm{TtS}$ & \# c. & cond & it & $\mathrm{TtS}$ \\
\hline FR4 & 1687 & $5.17 \mathrm{e}+1$ & 29 & $6.6 \mathrm{~s}$ & 9093 & $1.67 \mathrm{e}+2$ & 76 & $123.8 \mathrm{~s}$ \\
\hline $\mathrm{f}$ & 1687 & $2.52 \mathrm{e}+2$ & 94 & $14.2 \mathrm{~s}$ & 5061 & $1.19 \mathrm{e}+3$ & 274 & $275.6 s$ \\
\hline$f+r$ & - & - & - & - & 9093 & $5.09 \mathrm{e}+2$ & 179 & $211.7 \mathrm{~s}$ \\
\hline \multicolumn{9}{|c|}{ coefficient jump $1 \mathrm{e}+6 ; H / h=24$} \\
\hline coarse space & \# c. & cond & it & $\mathrm{TtS}$ & \# c. & cond & it & $\mathrm{TtS}$ \\
\hline FR4 & 1687 & $7.88 \mathrm{e}+1$ & 28 & $6.5 \mathrm{~s}$ & 9093 & $2.44 e+4$ & 179 & $210.9 \mathrm{~s}$ \\
\hline $\mathrm{f}$ & 1687 & $2.50 \mathrm{e}+5$ & 910 & $123.9 \mathrm{~s}$ & 5061 & $9.73 e+5$ & $>1000$ & $>893.7 \mathrm{~s}$ \\
\hline$f+r$ & - & - & - & - & 9093 & $4.70 \mathrm{e}+5$ & $>1000$ & $>924.9 \mathrm{~s}$ \\
\hline
\end{tabular}

5.5. Using an approximate coarse solver. Regardless which coarse space is chosen, solving the coarse problem with a sparse direct solver becomes a scaling bottleneck in all domain decomposition methods since the coarse space grows at least linearly with the number of subdomains. This bottleneck can be overcome in BDDC by approximating the coarse solve by, e.g., a recursive application of BDDC [64, 65] or an application of an algebraic multigrid (AMG) method [12, 36, 38]. Both approaches can be used in our BDDC implementation.

Here, we concentrate on the use of AMG (see [36] for a detailed discussion) and provide the results of a weak scaling experiment in Figure 5.8 for a diffusion problem. We always use BoomerAMG [29] from the hypre package with an aggressive HMIS (Hybrid Maximal Independent Set) coarsening and ext $i$ long range interpolation. As a coefficient distribution we again choose the shifted channels (see Figure 4.2) and a coefficient jump of 1e6. For 


\section{ETNA}

Kent State University and

Johann Radon Institute (RICAM)

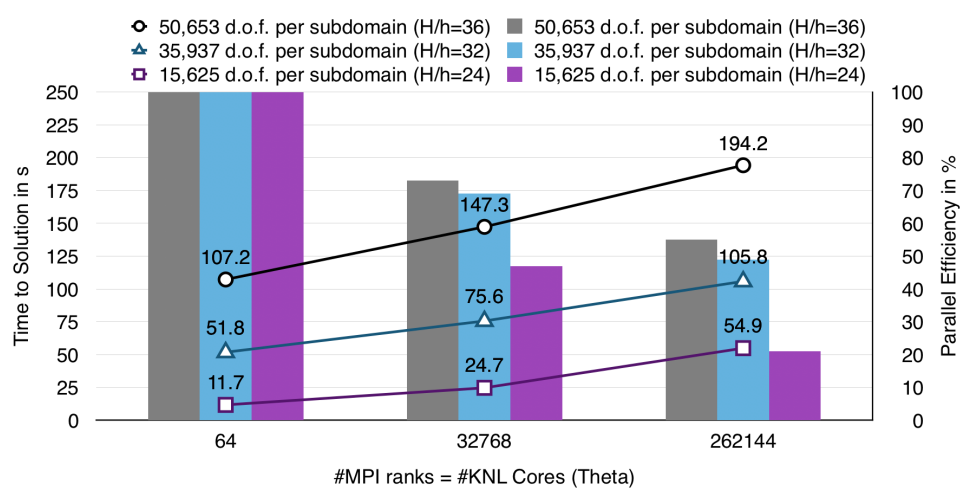

FIG. 5.8. Weak scalability of BDDC in three dimension using BoomerAMG to solve the FR4 coarse problem approximatively. Diffusion problem with a large coefficient of $\rho=1 \mathrm{e} 6$ inside shifted channels and 1 in the remaining domain; see Figure 4.2 for the coefficient distribution. Computed on Theta at Argonne National Laboratory, USA.

all tests, the number of CG iterations only varies between 18 and 22-also using 262144 subdomains on 262144 cores with a total problem size of more than 12 billion degrees of freedom $(H / h=36)$. Therefore, the coarse space FR4 is combinable with an approximate AMG solve without loosing robustness-at least for the considered coefficient distribution. For larger subdomain sizes, the scalability is satisfying with more than $55 \%$ parallel efficiency scaling from one KNL node to 4096 nodes.

6. Conclusions and future work. We have presented a frugal coarse space for FETI-DP and BDDC that does not require the solution of any local eigenvalue problems. FETI-DP and BDDC equipped with this new coarse space are robust for linear diffusion and linear elasticity tested for a broad variety of coefficient distributions. We showed this numerically considering many different coefficient distributions, including a realistic steel microstructure, and presented parallel weak scaling results for up to 262144 thousand subdomains and parallel tasks. We also heuristically motivate the robustness of the method using the local eigenvalue problems of adaptive FETI-DP or, equivalently, the bound on the $P_{D}$-operator well-known from the condition number estimate of FETI-DP and BDDC. A more refined theory will be subject of future research as well as an extension to a multilevel BDDC variant using our coarse space.

\section{REFERENCES}

[1] S. Badia, A. F. Martín, And H. NguYen, Physics-based balancing domain decomposition by constraints for multi-material problems, J. Sci. Comput., 79 (2019), pp. 718-747.

[2] S. BADIA, A. F. MARTín, AND J. PRINCIPE, On the scalability of inexact balancing domain decomposition by constraints with overlapped coarse/fine corrections, Parallel Comput., 50 (2015), pp. 1-24.

[3] - Multilevel balancing domain decomposition at extreme scales, SIAM J. Sci. Comput., 38 (2016), pp. C22-C52.

[4] L. Beirão da Veiga, L. F. PaVARino, S. Scacchi, O. B. Widlund, and S. Zampini, Isogeometric BDDC preconditioners with deluxe scaling, SIAM J. Sci. Comput., 36 (2014), pp. A1118-A1139.

[5] - Adaptive selection of primal constraints for isogeometric BDDC deluxe preconditioners, SIAM J. Sci. Comput., 39 (2017), pp. A281-A302.

[6] - Parallel sum primal spaces for isogeometric deluxe BDDC preconditioners, in Domain Decomposition Methods in Science and Engineering XXIII, C. Lee, C. Cai, D. E. Keyes, H. H. Kim, A. Klawonn, E. Park, and O. B. Widlund, eds., vol. 116 of Lect. Notes Comput. Sci. Eng., Springer, Cham, 2017, pp. 17-29.

[7] P. BJøRSTAD, J. Koster, AND P. KRZYZANOWSKI, Domain decomposition solvers for large scale industrial finite element problems, in Applied Parallel Computing. New Paradigms for HPC in Industry and Academia, T. Sørevik, F. Manne, A. H. Gebremedhin, and R. Moe, eds., vol. 1947 of Lecture Notes in Comput. Sci., Springer, Berlin, 2001, pp. 373-383. 
[8] D. Brands, D. Balzani, L. Scheunemann, J. Schröder, H. Richter, and D. RaAbe, Computational modeling of dual-phase steels based on representative three-dimensional microstructures obtained from EBSD data, Arch. Appl. Mech., 86 (2016), pp. 575-598.

[9] J. G. CALVO AND O. B. WIDLUND, An adaptive choice of primal constraints for BDDC domain decomposition algorithms, Electron. Trans. Numer. Anal., 45 (2016), pp. 524-544. http://etna.ricam.oeaw.ac.at/vol.45.2016/pp524-544.dir/pp524-544.pdf

[10] J.-M. CRos, A preconditioner for the Schur complement domain decomposition method, in Domain Decomposition Methods in Science and Engineering, O. W. I. Herrera, D. Keyes and R. Yates, eds., National Autonomous University of Mexico (UNAM), Mexico City, Mexico, 2003, pp. 373-380.

[11] C. R. DOHRMANN, A preconditioner for substructuring based on constrained energy minimization, SIAM J. Sci. Comput., 25 (2003), pp. 246-258.

[12] C. R. Dohrmann, An approximate BDDC preconditioner, Numer. Linear Algebra Appl., 14 (2007), pp. 149168.

[13] V. Dolean, F. NATAF, R. Scheichl, AND N. SPIllane, Analysis of a two-level Schwarz method with coarse spaces based on local Dirichlet-to-Neumann maps, Comput. Methods Appl. Math., 12 (2012), pp. 391-414.

[14] Y. EfEndiev, J. Galvis, R. LaZARov, AND J. Willems, Robust domain decomposition preconditioners for abstract symmetric positive definite bilinear forms, ESAIM Math. Model. Numer. Anal., 46 (2012), pp. 1175-1199.

[15] C. Farhat, M. Lesoinne, P. LeTallec, K. Pierson, And D. RiXen, FETI-DP: a dual-primal unified FETI method. I. A faster alternative to the two-level FETI method, Internat. J. Numer. Methods Engrg., 50 (2001), pp. 1523-1544.

[16] C. FARHAT, M. LeSoinne, AND K. Pierson, A scalable dual-primal domain decomposition method, Numer. Linear Algebra Appl., 7 (2000), pp. 687-714.

[17] J. GALVIS AND Y. EFENDIEv, Domain decomposition preconditioners for multiscale flows in high-contrast media, Multiscale Model. Simul., 8 (2010), pp. 1461-1483.

[18] _ Domain decomposition preconditioners for multiscale flows in high contrast media: reduced dimension coarse spaces, Multiscale Model. Simul., 8 (2010), pp. 1621-1644.

[19] M. J. GANDER, A. LONELAND, AND T. RAHMAN, Analysis of a new harmonically enriched multiscale coarse space for domain decomposition methods, Preprint on arXiv, 2015. https://arxiv.org/abs/1512.05285

[20] S. GiPPERT, A. KLAWONn, AND O. RHEINBACH, Analysis of FETI-DP and BDDC for linear elasticity in $3 D$ with almost incompressible components and varying coefficients inside subdomains, SIAM J. Numer. Anal., 50 (2012), pp. 2208-2236.

[21] _ A deflation based coarse space in dual-primal FETI methods for almost incompressible elasticity, in Numerical Mathematics and Advanced Applications-ENUMATH 2013, A. Abdulle, S. Deparis, D. Kressner, F. Nobile, and M. Picasso, eds., vol. 103 of Lect. Notes Comput. Sci. Eng., Springer, Cham, 2015, pp. 573-581.

[22] A. HeInLEIN, Parallel Overlapping Schwarz Preconditioners and Multiscale Discretizations with Applications to Fluid-Structure Interaction and Highly Heterogeneous Problems, PhD. Thesis, Department Mathematik, Universität zu Köln, Cologne, 2016.

[23] A. Heinlein, A. Klawonn, J. Knepper, And O. Rheinbach, An adaptive GDSW coarse space for two-level overlapping Schwarz methods in two dimensions, in Domain Decomposition Methods in Science and Engineering XXIV, P. E. Bjørstad, S. C. Brenner, L. Halpern, H. H. Kim, R. Kornhuber, T. Rahman, and O. B. Widlund, eds., vol. 125 of Lect. Notes Comput. Sci. Eng., Springer, Cham, 2018, pp. 373-382.

[24] - Multiscale coarse spaces for overlapping Schwarz methods based on the ACMS space in 2D, Electron. Trans. Numer. Anal., 48 (2018), pp. 156-182.

http://etna.ricam.oeaw.ac.at/vol.48.2018/pp156-182.dir/pp156-182.pdf

[25] - Adaptive GDSW coarse spaces for overlapping Schwarz methods in three dimensions, SIAM J. Sci. Comput., 41 (2019), pp. A3045-A3072.

[26] A. Heinlein, A. KLAWONN, AND M. J. KÜHN, Local spectra of adaptive domain decomposition methods, in Domain Decomposition Methods in Science and Engineering XXV, R. Haynes, S. MacLachlan, X.-C. Cai, L. Halpern, H. H. Kim, A. Klawonn, and O. Widlund, eds., vol. 138 of Lect. Notes Comput. Sci. Eng., Springer Cham, 2020, pp. 167-175.

[27] A. Heinlein, A. Klawonn, M. Lanser, And J. Weber, Machine learning in adaptive domain decomposition methods—predicting the geometric location of constraints, SIAM J. Sci. Comput., 41 (2019), pp. A3887-A3912.

[28] - Machine learning in adaptive FETI-DP - a comparison of smart and random training data, in Domain Decomposition Methods in Science and Engineering XXV, R. Haynes, S. MacLachlan, X.-C. Cai, L. Halpern, H. H. Kim, A. Klawonn, and O. Widlund, eds., vol. 138 of Lect. Notes Comput. Sci. Eng., Springer, Cham, 2020, pp. 218-226. 
[29] V. E. Henson And U. M. YAng, V. E. Henson And U. M. YAng, BoomerAMG: A parallel algebraic multigrid solver and preconditioner, Appl. Num. Math., 41 (2002), pp. 155-177.

[30] H. H. KIM AND E. T. CHUNG, A BDDC algorithm with enriched coarse spaces for two-dimensional elliptic problems with oscillatory and high contrast coefficients, Multiscale Model. Simul., 13 (2015), pp. 571593.

[31] A. KLAWOnN, M. KÜHn, AND O. RHEINBACH, Adaptive coarse spaces for FETI-DP in three dimensions, SIAM J. Sci. Comput., 38 (2016), pp. A2880-A2911.

[32] _ - Adaptive coarse spaces for FETI-DP in three dimensions with applications to heterogeneous diffusion problems, in Domain Decomposition Methods in Science and Engineering XXIII, C. Lee, C. Cai, D. E. Keyes, H. H. Kim, A. Klawonn, E. Park, and O. B. Widlund, eds., vol. 116 of Lect. Notes Comput. Sci. Eng., Springer, Cham, 2017, pp. 187-196.

[33] - Adaptive FETI-DP and BDDC methods with a generalized transformation of basis for heterogeneous problems, Electron. Trans. Numer. Anal., 49 (2018), pp. 1-27.

http://etna.ricam.oeaw.ac.at/vol.49.2018/pp1-27.dir/pp1-27.pdf

[34] A. Klawonn, M. LANSER, AND O. RHeInBACH, Toward extremely scalable nonlinear domain decomposition methods for elliptic partial differential equations, SIAM J. Sci. Comput., 37 (2015), pp. C667-C696.

[35] — A highly scalable implementation of inexact nonlinear FETI-DP without sparse direct solvers, in Numerical Mathematics and Advanced Applications-ENUMATH 2015, B. Karasözen, M. Manguoglu, M. Tezer-Sezgin, S. Göktepe, and Ö. Ugur, eds., vol. 112 of Lect. Notes Comput. Sci. Eng., Springer, Cham, 2016, pp. 255-264.

[36] — Nonlinear BDDC methods with approximate solvers, Electron. Trans. Numer. Anal., 49 (2018), pp. 244-273.

http://etna.ricam.oeaw.ac.at/vol.49.2018/pp244-273.dir/pp244-273.pdf

[37] A. Klawonn, M. Lanser, O. RheinbaCh, And M. Uran, Nonlinear FETI-DP and BDDC methods: a unified framework and parallel results, SIAM J. Sci. Comput., 39 (2017), pp. C417-C451.

[38] A. Klawonn, M. Lanser, O. Rheinbach, AND J. Weber, Preconditioning the coarse problem of BDDC methods-three-level, algebraic multigrid, and vertex-based preconditioners, Electron. Trans. Numer. Anal., 51 (2019), pp. 432-450.

http://etna.ricam.oeaw.ac.at/vol.51.2019/pp432-450.dir/pp432-450.pdf

[39] A. KLAWOnN, P. RADTKE, AND O. RHEINBACH, FETI-DP methods with an adaptive coarse space, SIAM J. Numer. Anal., 53 (2015), pp. 297-320.

[40] - A comparison of adaptive coarse spaces for iterative substructuring in two dimensions, Electron. Trans. Numer. Anal., 45 (2016), pp. 75-106.

http://etna.ricam.oeaw.ac.at/vol.45.2016/pp75-106.dir/pp75-106.pdf

[41] A. KLAWONN AND O. RHEINBACH, A parallel implementation of dual-primal FETI methods for threedimensional linear elasticity using a transformation of basis, SIAM J. Sci. Comput., 28 (2006), pp. 18861906.

[42] - Robust FETI-DP methods for heterogeneous three dimensional elasticity problems, Comput. Methods Appl. Mech. Engrg., 196 (2007), pp. 1400-1414.

[43] _ Highly scalable parallel domain decomposition methods with an application to biomechanics, ZAMM Z. Angew. Math. Mech., 90 (2010), pp. 5-32.

[44] - Deflation, projector preconditioning, and balancing in iterative substructuring methods: connections and new results, SIAM J. Sci. Comput., 34 (2012), pp. A459-A484.

[45] A. KLAWOnN, O. RheINBACH, AND O. B. WIDLUnd, An analysis of a FETI-DP algorithm on irregular subdomains in the plane, SIAM J. Numer. Anal., 46 (2008), pp. 2484-2504.

[46] A. Klawonn And O. B. Widlund, Dual-primal FETI methods for linear elasticity, Comm. Pure Appl. Math., 59 (2006), pp. 1523-1572.

[47] _ _ Dual-primal FETI methods for linear elasticity, Comm. Pure Appl. Math., 59 (2006), pp. 1523-1572.

[48] A. KLaWonn, O. B. WIDLund, AND M. DRYJA, Dual-primal FETI methods for three-dimensional elliptic problems with heterogeneous coefficients, SIAM J. Numer. Anal., 40 (2002), pp. 159-179.

[49] J. KNEPPER, Multiskalen-Grobgitterräume für Überlappende Schwarz-Gebietszerlegungsverfahren, Master Thesis, University of Cologne, Köln, 2016.

[50] J. Li AND O. B. WidLund, FETI-DP, BDDC, and block Cholesky methods, Internat. J. Numer. Methods Engrg., 66 (2006), pp. 250-271.

[51] - On the use of inexact subdomain solvers for BDDC algorithms, Comput. Methods Appl. Mech. Engrg., 196 (2007), pp. 1415-1428.

[52] J. Mandel And C. R. Dohrmann, Convergence of a balancing domain decomposition by constraints and energy minimization, Numer. Linear Algebra Appl., 10 (2003), pp. 639-659.

[53] J. Mandel, C. R. Dohrmann, AND R. TEZAur, An algebraic theory for primal and dual substructuring methods by constraints, Appl. Numer. Math., 54 (2005), pp. 167-193. 
[54] J. MAndel And B. Sousedík, Adaptive selection of face coarse degrees of freedom in the BDDC and the FETI-DP iterative substructuring methods, Comput. Methods Appl. Mech. Engrg., 196 (2007), pp. 1389-1399.

[55] J. MANdel, B. Sousedík, AND J. Sístek, Adaptive BDDC in three dimensions, Math. Comput. Simulation, 82 (2012), pp. 1812-1831.

[56] J. MANDEL AND R. TEZAUR, On the convergence of a dual-primal substructuring method, Numer. Math., 88 (2001), pp. 543-558.

[57] D.-S. OH, O. B. WidLund, S. ZAMPINI, AND C. R. Dohrmann, BDDC algorithms with deluxe scaling and adaptive selection of primal constraints for Raviart-Thomas vector fields, Math. Comp., 87 (2018), pp. 659-692.

[58] C. PeChstein And C. R. Dohrmann, A unified framework for adaptive BDDC, Electron. Trans. Numer. Anal., 46 (2017), pp. 273-336.

http://etna.ricam.oeaw.ac.at/vol.46.2017/pp273-336.dir/pp273-336.pdf

[59] C. PECHSTEIN AND R. SCHEICHL, Analysis of FETI methods for multiscale PDEs, Numer. Math., 111 (2008), pp. 293-333.

[60] P. RADTKE, Adaptive Coarse Spaces for FETI-DP and BDDC Methods, PhD. Thesis, Department Mathematik, Universität zu Köln, Cologne, 2015.

[61] N. Spillane, V. Dolean, P. Hauret, F. Nataf, C. Pechstein, and R. Scheichl, Abstract robust coarse spaces for systems of PDEs via generalized eigenproblems in the overlaps, Numer. Math., 126 (2014), pp. 741-770.

[62] N. SPILlane AND D. J. RiXen, Automatic spectral coarse spaces for robust finite element tearing and interconnecting and balanced domain decomposition algorithms, Internat. J. Numer. Methods Engrg., 95 (2013), pp. 953-990.

[63] A. Toselli And O. Widlund, Domain Decomposition Methods, Springer, Berlin, 2005.

[64] X. TU, Three-level BDDC in three dimensions, SIAM J. Sci. Comput., 29 (2007), pp. 1759-1780.

[65] — Three-level BDDC in two dimensions, Internat. J. Numer. Methods Engrg., 69 (2007), pp. 33-59.

[66] S. ZAMPINI, PCBDDC: a class of robust dual-primal methods in PETSc, SIAM J. Sci. Comput., 38 (2016), pp. S282-S306. 